日本原子力研究開発機構機関リポジトリ

Japan Atomic Energy Agency Institutional Repository

\begin{tabular}{|c|l|}
\hline Title & $\begin{array}{l}\text { Quantum optimal control of the isotope-selective rovibrational } \\
\text { excitation of diatomic molecules }\end{array}$ \\
\hline Author(s) & Kurosaki Yuzuru, Yokoyama Keiichi \\
\hline Citation & Chemical Physics, 493, p.183-193 (2017) \\
\hline Text Version & Author Accepted Manuscript \\
\hline URL & $\underline{\text { https://jopss.jaea.go.jp/search/servlet/search?5059879 }}$ \\
\hline DOI & $\underline{\text { https://doi.org/10.1016/j.chemphys.2017.07.003 }}$ \\
\hline Right & $\begin{array}{l}\text { C } 2017 . \text { This manuscript version is made available under the } \\
\text { CC-BY-NC-ND 4.0 license } \\
\text { http://creativecommons.org/licenses/by־nc-nd/4.0/ }\end{array}$ \\
\hline
\end{tabular}




\title{
Quantum optimal control of the isotope-selective rovibrational excitation of diatomic molecules
}

\author{
Yuzuru Kurosaki ${ }^{a}$ and Keiichi Yokoyama ${ }^{b}$
}

${ }^{a}$ Quantum Beam Science Research Directorate, Takasaki Advanced Radiation Research Institute, National Institutes for Quantum and Radiological Science and Technology, Tokai, Ibaraki 319-1195, Japan,

${ }^{\mathrm{b}}$ Materials Science Research Center, Japan Atomic Energy Agency, 1-1-1 Sayo-cho, Sayo-gun, Hyogo 679-5148, Japan

\section{ABSTRACT}

We carry out optimal control theory calculations for isotope-selective pure rotational and vibrational-rotational excitations of diatomic molecules. The fifty-fifty mixture of diatomic isotopologues, ${ }^{7} \mathrm{Li}^{37} \mathrm{Cl}$ and ${ }^{7} \mathrm{Li}^{35} \mathrm{Cl}$, is considered and the molecules are irradiated with a control pulse. In the wave packet propagation we employ the method quantum mechanically rigorous for the two-dimensional system including both the radial and angular motions. We investigate quantum controls of the isotope-selective pure rotational excitation for two total times 1280000 and 2560000 a.u. (31.0 and 61.9 ps) and the vibrational-rotational excitation for three total times, 640000, 1280000, and 2560000 a.u. $(15.5,31.0$, and $61.9 \mathrm{ps})$ The initial state is set to that both the isotopologues are in the ground vibrational and rotational levels, $v=0$ and $J=0$. The target state for pure rotational excitation is set to ${ }^{7} \mathrm{Li}^{37} \mathrm{Cl}(v=0, J=1)$ and ${ }^{7} \mathrm{Li}^{35} \mathrm{Cl}(v=0, J=0)$; that for vibrational-rotational excitation is set to ${ }^{7} \mathrm{Li}^{37} \mathrm{Cl}(v=1, J=1)$ and ${ }^{7} \mathrm{Li}^{35} \mathrm{Cl}(v=0, J=0)$. The obtained final yields are quite high and those for the longest total time are calculated to be nearly 1.0. When total time is 1280000 a.u., the final yields for the pure rotational excitation are slightly smaller than those for the vibrational-rotational excitation. This is because the isotope shift (difference in transition energy between the two isotopologues) for the pure rotational transition between low-lying levels is much smaller than that for the vibrational-rotational transition. We thus theoretically succeed in controlling the isotope-selective excitations of diatomic molecules using the method including both radial and angular motions quantum mechanically. 
Keywords:

Quantum control

Optimal control theory

Isotope-selective rovibrational excitation

Laser pulse

Diatomic molecule

Wave packet

E-mail addresses: kurosaki.yuzuru@ qst.go.jp (Y. Kurosaki); yokoyama.keiichi@jaea.go.jp (K. Yokoyama). 


\section{Introduction}

Quantum control has been expected to bring a breakthrough not only in basic science but also in applied research leading to novel industrial technology. Thus a great number of studies has been devoted to quantum control for more than two decades [1-4]. One of the most important applications of quantum control is the discrimination of a mixture of physically or chemically similar species, where different species are driven into different target states at the same time with a single laser pulse. Rabitz and co-workers [5-7] presented the formulation called optimal dynamic discrimination (ODD), which is a theoretical tool to predict a control laser pulse to enable such discrimination of a mixture of similar quantum systems. It is noteworthy that some experimental demonstrations of ODD have been reported, such as the isotope-selective ionization of diatomic molecules $[8,9]$ and the discrimination of competitive product channels for large biomolecules $[10,11]$.

We [12-15] have studied, using optimal control theory (OCT) [16-18], the quantum optimal control of isotope-selective vibrational excitations of a diatomic alkali halide. As a result of the numerical simulations, ODD for a mixture was successfully demonstrated; it was verified that the obtained optimal field drives the system wave packets of different initial states into different final states. In those studies molecular rotational motion was frozen and only the radial (vibrational) motion was considered.

On the other hand, we [19-22] are also interested in isotope-selective rotational excitations of diatomic alkali halides using an optical frequency comb. This is because in the current laser shaping technology the design of the frequency comb for rotational excitations of heavy diatomic molecules is more realistic than that of the laser pulse for vibrational excitations. Very recently, we [23] have proposed a method of the isotopeselective rovibrational excitation for a gas-phase mixture of diatomic alkali halides at a finite temperature using a laser pulse consisting of a frequency comb for rotational excitations and a subsequent bunch of pulse for vibrational excitations; it is expected that the frequency comb tuned to the rotational transition energies of one isotopologue changes the rotational-state distributions and magnifies the difference in vibrational transition energies between the isotopologues, thus enabling the isotope-selective vibrational excitation even with the single frequency pulse. The numerical simulation has shown that the proposed scheme works well for the isotope-selective rovibrational 
excitation for a gas-phase mixture of the diatomic ${ }^{7} \mathrm{Li}^{37} \mathrm{Cl}$ and ${ }^{7} \mathrm{Li}^{35} \mathrm{Cl}$ molecules at $70 \mathrm{~K}$.

In the present study we perform OCT simulations to obtain optimal electric fields that enable the isotope-selective rovibrational excitation for the isotopologues: ${ }^{7} \mathrm{Li}^{37} \mathrm{Cl}$ and ${ }^{7} \mathrm{Li}^{35} \mathrm{Cl}$. In the wave-packet calculations both the radial (vibrational) and angular (rotational) motions are treated quantum mechanically; this is more challenging than our previous studies [16-18] mentioned above, where only the radial motion was considered. The ultimate goal might be the control of a mixed ensemble of molecules thermally distributed up to high quantum levels according to the temperature of the ensemble. We focus here, however, on the simplest cases of the isotope-selective excitations and examine the following two excitations: (i) pure rotational excitation; (ii) vibrationalrotational excitation. In the two cases the initial state is set to the condition that both ${ }^{7} \mathrm{Li}^{37} \mathrm{Cl}$ and ${ }^{7} \mathrm{Li}^{35} \mathrm{Cl}$ are in the ground vibrational and rotational levels, $v=0$ and $J=0$.

The target states in these cases are set as follows: (i) ${ }^{7} \mathrm{Li}^{37} \mathrm{Cl}(v=0, J=1),{ }^{7} \mathrm{Li}^{35} \mathrm{Cl}(v=0$, $J=0)$; (ii) ${ }^{7} \mathrm{Li}^{37} \mathrm{Cl}(v=1, J=1),{ }^{7} \mathrm{Li}^{35} \mathrm{Cl}(v=0, J=0)$.

Theoretical details are described in Sec. II. The results of calculation are presented in Sec. III and conclusions are given in Sec. IV.

\section{Theoretical details}

\subsection{OCT for isotope-selective excitation}

OCT provides an efficient tool to find an electric field $\varepsilon(t)$ that causes the transition between predetermined initial and target states with a high probability. In the present study a gas-phase mixture of isotopologues is considered and the objective functional $J$ to be maximized is given as

$$
\begin{aligned}
J= & \sum_{A} p_{A}\left\langle\psi_{A}(T)\left|O_{A}\right| \psi_{A}(T)\right\rangle-\alpha \int_{0}^{T} d t \varepsilon(t)^{2} \\
& -2 \sum_{A} p_{A} \operatorname{Re}\left[\int_{0}^{T} d t\left\langle\chi_{A}(t)\left|\frac{\partial}{\partial t}+i\left(H_{0}^{A}-\mu \varepsilon(t)\right)\right| \psi_{A}(t)\right\rangle\right] .
\end{aligned}
$$

The first term is the transition probability to be maximized and is referred to as final yield, where $p_{A}$ is the population of isotopologue $\mathrm{A}, \psi_{A}(t)$ is the wave packet of isotopologue $\mathrm{A}$ propagated from time $(t)=0$ to $T$, and $O_{A}$ is the target operator of isotopologue A. The 
second term is the laser fluence to be minimized, where $\alpha$ is a penalty factor. In order to obtain the optimal fields that start and end with the zero amplitude, a time dependence is introduced in $\alpha$.

$$
\alpha=\alpha(t)=\frac{\alpha_{0}}{s(t)}
$$

where $\alpha_{0}$ is a positive constant and $s(t)$ is a shape function having a form

$$
s(t)=\sin ^{2}\left(\frac{\pi t}{T}\right) .
$$

The third term is the dynamical constraint, where $\chi_{A}(t)$ is the Lagrange multiplier of isotopologue $\mathrm{A}$, Re denotes the real part of a complex number, $H_{0}{ }^{A}$ is the field-free Hamiltonian of isotopologue $\mathrm{A}$, and $\mu$ is the dipole moment. In the present OCT calculations we consider the $1: 1$ mixture of ${ }^{7} \mathrm{Li}^{37} \mathrm{Cl}(\mathrm{A})$ and ${ }^{7} \mathrm{Li}^{35} \mathrm{Cl}(\mathrm{B})$ and $p_{A}=p_{B}=0.5$.

Varying the objective functional $J$ with respect to $\psi_{A}(t), \chi_{A}(t)$, and $\varepsilon(t)$, we obtain the following three coupled equations for each isotopologue:

$$
\begin{aligned}
& i \frac{\partial \psi_{A}(t)}{\partial t}=\left(H_{0}^{A}-\mu \varepsilon(t)\right) \psi_{A}(t), \quad \psi_{A}(0)=\psi_{A, 0} \\
& i \frac{\partial \chi_{A}(t)}{\partial t}=\left(H_{0}^{A}-\mu \varepsilon(t)\right) \chi_{A}(t), \quad \chi_{A}(T)=O \psi_{A}(T) \\
& \varepsilon(t)=-\frac{s(t)}{\alpha_{0}} \sum_{A} p_{A} \operatorname{Im}\left\langle\chi_{A}(t)|\mu| \psi_{A}(t)\right\rangle,
\end{aligned}
$$

with Im in Eq. (4c) denoting the imaginary part. These equations are iteratively solved until the value of $J$ is converged using the monotonically convergent algorithm [24], i.e., the optimization of $J$ is achieved by iteratively solving the following equations at the $k$ th step:

$$
\begin{aligned}
& i \frac{\partial \psi_{A}^{k}(t)}{\partial t}=\left(H_{0}^{A}-\mu \varepsilon^{k}(t)\right) \psi_{A}^{k}(t), \quad \psi_{A}^{k}(0)=\psi_{A, 0} \\
& \varepsilon^{k}(t)=(1-\delta) \tilde{\varepsilon}^{k-1}(t)-\frac{\delta s(t)}{\alpha_{0}} \sum_{A} p_{A} \operatorname{Im}\left\langle\chi_{A}^{k-1}(t)|\mu| \psi_{A}^{k}(t)\right\rangle \\
& i \frac{\partial \chi_{A}^{k}(t)}{\partial t}=\left(H_{0}^{A}-\mu \tilde{\varepsilon}^{k}(t)\right) \chi_{A}^{k}(t), \quad \chi_{A}^{k}(T)=O \psi_{A}^{k}(T) \\
& \tilde{\varepsilon}^{k}(t)=(1-\eta) \varepsilon^{k}(t)-\frac{\eta s(t)}{\alpha_{0}} \sum_{A} p_{A} \operatorname{Im}\left\langle\chi_{A}^{k}(t)|\mu| \psi_{A}^{k}(t)\right\rangle .
\end{aligned}
$$

\subsection{Wave packet propagation}


The time-dependent Schrödinger equation for the wave packet $\psi(t)$ of nucleus, in the Born-Oppenheimer framework, is given by

$$
i \frac{\partial}{\partial t} \psi(t)=H \psi(t)
$$

where the total Hamiltonian $H$ is the sum of the field-free and the light-molecule interaction parts:

$$
H=H_{0}+H_{I} \text {. }
$$

The field-free Hamiltonian $H_{0}$ is composed of the kinetic energy operators for the radial distance $R$, the polar angle $\theta$, and the azimuth angle $\phi$, and of the potential energy operator $V(R)$ :

$$
H_{0}=T_{R}+T_{\theta}+T_{\phi}+V(R) .
$$

In a previous work [25] potential energy curves for the $\mathrm{LiCl}$ molecule were calculated using high-level ab initio methods and here the calculated ground $\mathrm{X}^{1} \Sigma^{+}$state potential energy is employed for $V(R)$. The kinetic energy operators are given by

$$
\begin{aligned}
& T_{R}=-\frac{1}{2 m R^{2}} \frac{\partial}{\partial R}\left(R^{2} \frac{\partial}{\partial R}\right) \\
& T_{\theta}=-\frac{1}{2 m R^{2}} \frac{1}{\sin \theta} \frac{\partial}{\partial \theta}\left(\sin \theta \frac{\partial}{\partial \theta}\right) \\
& T_{\phi}=-\frac{1}{2 m R^{2}} \frac{1}{\sin ^{2} \theta} \frac{\partial^{2}}{\partial \phi^{2}},
\end{aligned}
$$

where $m$ is the reduced mass. $H_{I}$ is the field-molecule interaction term and is given by

$$
H_{I}=-\mu(R) \varepsilon(t) \cos \theta \text {. }
$$

The ground $\mathrm{X}^{1} \Sigma^{+}$state dipole moment calculated in the previous work [25] is employed for $\mu(R)$. In the present calculation the system is assumed to interact with the electric field only through the permanent dipole and the higher-order terms of the electric field are neglected. This might be justified because the obtained electric fields are not so strong, as shown later; it is of interest, however, to investigate interaction through the higherorder terms and this is a future challenge. As usual, the eigenfunction for $H_{0}$ can be factorized into the three factors depending on $R, \theta$, and $\phi$, and thus the initial wave packet is set to an eigenfunction: 


$$
\psi(0)=\chi_{v J}(R) Y_{J M_{J}}(\theta, \phi)
$$

where $Y_{J M J}(\theta, \phi)$ is the spherical harmonics and $\chi_{v J}(R)$ is the eigenfunction of the vibrational state $v$ obtained by solving the following $J$-dependent radial Schrödinger equation:

$$
\left[-\frac{1}{2 m} \frac{\partial^{2}}{\partial R^{2}}+V(R)+\frac{1}{2 m R^{2}} J(J+1)\right] \chi_{v J}(R)=E_{v J} \chi_{v J}(R) .
$$

This equation is numerically solved using the Fourier grid Hamiltonian method [26]. In the present calculations we only consider the $M_{J}=0$ case and thus Eq. (13) reduces to

$$
\psi(0)=\chi_{v J}(R) \sqrt{(2 J+1) / 2} P_{J}^{0}(\cos \theta),
$$

where $P_{J}^{0}$ is associated Legendre polynomials for $M_{J}=0$. Eq. (3) is numerically integrated using the split-operator method [27-29]:

$$
\begin{aligned}
\psi(t+\Delta t)= & \exp \left[-i \Delta t\left(T_{R}+T_{\theta}+V+H_{I}\right)\right] \psi(t) \\
\approx & \exp \left(-i\left(V+H_{I}\right) \Delta t / 2\right) \exp \left(-i T_{\theta} \Delta t / 2\right) \exp \left(-i T_{R} \Delta t\right) \\
& \quad \times \exp \left(-i T_{\theta} \Delta t / 2\right) \exp \left(-i\left(V+H_{I}\right) \Delta t / 2\right) \psi(t)+O\left(\Delta t^{3}\right)
\end{aligned}
$$

The radial grid points are chosen to be evenly spaced and the angular grid points are chosen as the Gauss-Legendre quadrature points [30]. The action of the potential and field-molecule interaction $V+H_{I}$ on the wave packet is performed directly in coordinate space by multiplication. The kinetic-energy operator $T_{R}$ is acted on the wave packet in momentum space by transforming from coordinate space with the fast-Fourier-transform (FFT) algorithm, and then the wave packet is transformed back into the coordinate space with the reverse FFT algorithm. The kinetic-energy operator $T_{\theta}$ is acted on the wave packet in the polynomial representation by switching from coordinate space, and then the wave packet is switched back into coordinate space, using the discrete variable representation (DVR) technique [31]. To eliminate the artificial reflection of the wave packet at the edge of radial grid points, we incorporate a damping function [32] that is operative only for the last five points from the edge. Grid and time parameters used in the present OCT calculations are summarized in Table I.

\section{Results and discussion}

In the present study we investigate two total times ( $T$ 's), 1280000 and 2560000 a.u. 
(31.0 and $61.9 \mathrm{ps),} \mathrm{for} \mathrm{pure} \mathrm{rotational} \mathrm{excitation} \mathrm{and} \mathrm{three} T$ 's, 640000, 1280000, and 2560000 a.u. (15.5, 31.0, and $61.9 \mathrm{ps})$, for vibrational-rotational excitation. The employed initial guess for the optimal field for pure rotational excitation is just $\varepsilon(t)=E_{0} \cos 2 B t$, where $B$ is the rotational constant for ${ }^{7} \mathrm{Li}^{37} \mathrm{Cl}$ and $E_{0}=5.0 \times 10^{-4}$ and $1.0 \times 10^{-5}$ a.u. for $T$ $=1280000$ and 2560000 a.u., respectively. The employed initial guess field for vibrational-rotational excitation is $\varepsilon(t)=E_{0} \cos \omega_{0} t$, where $\omega_{0}$ is the transition energy between the $v=0$ and $J=0$ state and the $v=1$ and $J=1$ state for ${ }^{7} \mathrm{Li}^{37} \mathrm{Cl}$ and $E_{0}=1.0 \mathrm{x}$ $10^{-3}, 5.0 \times 10^{-4}$, and $1.0 \times 10^{-5}$ a.u. for $T=640000,1280000$, and 2560000 a.u., respectively.

\subsection{Rotational excitation}

In this subsection the results of OCT calculations for the isotope-selective rotational excitation, ${ }^{7} \mathrm{Li}{ }^{37} \mathrm{Cl}(v=0, J=0) ;{ }^{7} \mathrm{Li}^{35} \mathrm{Cl}(v=0, J=0) \rightarrow{ }^{7} \mathrm{Li}^{37} \mathrm{Cl}(v=0, J=1) ;{ }^{7} \mathrm{Li}^{35} \mathrm{Cl}(v$ $=0, J=0)$, are presented. It was found that without filtering the control field frequency the OCT calculations often yield the optimal fields containing a zero frequency component, which is experimentally unrealistic. Thus the low/high cutoff frequencies $\omega_{l}$ and $\omega_{h}$ are set up and frequencies lower than $\omega_{l}$ and those higher than $\omega_{h}$ are filtered out in the OCT calculation. Here we adopt two sets of cutoffs: (i) $\omega_{l}=6.0 \times 10^{-6}$ a.u. and $\omega_{h}$ $=0.1$ a.u.; (ii) $\omega_{l}=6.0 \times 10^{-6}$ a.u. and $\omega_{h}=1.0 \times 10^{-3}$ a.u. Frequency cutoff (i) allows both rotational and vibrational transitions but (ii) allows only rotational transition.

\subsection{1. $T=1280000$ a.u.}

First, the OCT result obtained with frequency cutoff (i) is presented. The final yield is 0.903 , the maximum field amplitude $3.014 \times 10^{-3}$ a.u., and the fluence $2.606 \times 10^{-1}$ a.u., Table II. Figure 1a shows the optimal field as a function of $t$ and Fig. 1b its spectrum. As shown in Fig. 1b, the spectrum is mostly composed of three components; the fundamental, the first overtone, and the second overtone for the vibrational transition. The first overtone has the largest contribution, the fundamental the second, and the second overtone the smallest. Inset in Fig. 1b shows a close-up of the spectrum in a low-frequency region; although contribution is small, several frequency components are seen around $5 \times 10^{-5}$ 
a.u., corresponding to rotational transitions. In order to make sure if those components play a role in the control mechanism frequencies lower than $4 \times 10^{-4}$ a.u. were cut out from the optimal field and the final yield was again calculated. As a result, the yield dropped to less than 0.5 , indicating that the lower-frequency components play a significant role. Figure 2 shows temporal changes in the populations of vibrational and rotational states for the two isotopologues, caused by the obtained optimal field. The timedependent population of vibrational state $v, p_{v}(t)$, is given by the summation, over the number of angular grid point $N_{a}$, of the square of the projection of the rovibrational eigenfunction onto the system wave packet $\psi(t)$ :

$$
p_{v}(t)=\sum_{J}^{N a}\left|\left\langle\psi(t) \mid \chi_{v J}(R) \sqrt{(2 J+1) / 2} P_{J}^{0}(\cos \theta)\right\rangle\right|^{2}
$$

and that of rotational state $J, p_{J}(t)$, is given by the summation, over the number of radial grid point $N_{r}$, of the same quantity:

$$
p_{J}(t)=\sum_{v}^{N r}\left|\left\langle\psi(t) \mid \chi_{v J}(R) \sqrt{(2 J+1) / 2} P_{J}^{0}(\cos \theta)\right\rangle\right|^{2} .
$$

At the beginning of the control pulse the populations of the vibrational $v=0$ state and the rotational $J=0$ state are 1.0 for both ${ }^{7} \mathrm{Li}^{37} \mathrm{Cl}$ and ${ }^{7} \mathrm{Li}^{35} \mathrm{Cl}$. As shown in Figs. 2a and 2 b, in both the isotopologues vibrational states are excited up to $v=4$ around the middle of the pulse and finally the population of the $v=0$ state is more than 0.9 at the end. The rotational states, Figs. 2c and 2d, show similar behaviors to the vibrational-state counterparts and they are excited up to $J=5$, although the populations change very rapidly. Finally, the populations of the target states, $J=1\left({ }^{7} \mathrm{Li}^{37} \mathrm{Cl}\right)$ and $J=0\left({ }^{7} \mathrm{Li}^{35} \mathrm{Cl}\right)$, reach more than 0.9 .

Next, the OCT result obtained with frequency cutoff (ii) is discussed. The final yield is 0.921 , the maximum field amplitude $6.552 \times 10^{-4}$ a.u., and the fluence $6.201 \times 10^{-2}$ a.u., Table II. Although the final yield is comparable with that with frequency cutoff (i), the field intensity is much smaller. Figure 3a shows the optimal field and Fig. $3 \mathrm{~b}$ its spectrum. The optimal field changes very slowly with small rapid oscillations. The spectrum is mainly composed of frequency components around $1.0 \times 10^{-5}$ a.u. with many other components being distributed over a wide frequency range. Inset in Fig. $3 \mathrm{~b}$ shows a closeup of the spectrum around $1.0 \times 10^{-5}$ a.u., where filled circles are used to indicate the discrete points at intervals of the minimum frequency. The figure reveals that the frequency component of the largest contribution lies around $1.3 \times 10^{-5}$ a.u. and that of the 
second largest contribution around $7.0 \times 10^{-6}$ a.u.; the former is close to the rotational $J=$ $2 \leftarrow 1$ transition energy of ${ }^{7} \mathrm{Li}^{37} \mathrm{Cl}$ equal to $4 B=1.29 \times 10^{-5}$ a.u. and the latter to the $J=1$ $\leftarrow 0$ transition energy equal to $2 B=6.45 \times 10^{-6}$ a.u. Figure 4 shows temporal changes in the populations of vibrational and rotational states for the two isotopologues. No vibrational excitation occurs by filtering out frequencies larger than $1.0 \times 10^{-3}$ a.u., Figs. $4 \mathrm{a}$ and $4 \mathrm{~b}$. Rotational states are actively excited up to around $J=12$, Figs. $4 \mathrm{c}$ and $4 \mathrm{~d}$, and finally the target states, $J=1\left({ }^{7} \mathrm{Li}^{37} \mathrm{Cl}\right)$ and $J=0\left({ }^{7} \mathrm{Li}^{35} \mathrm{Cl}\right)$, are achieved at a transition probability higher than 0.9 .

The minimum frequency $\omega_{\min }$ that can be represented by the pulse of total time $T$ is given by $2 \pi / T$ and for the present pulse of $T=1280000$ a.u. $\omega_{\min }=4.9 \times 10^{-6}$ a.u. The rotational transition energy from $J=0$ to $J=1$ given by $2 B$ are $6.45 \times 10^{-6}$ and $6.51 \times 10^{-}$ ${ }^{6}$ a.u., respectively, for ${ }^{7} \mathrm{Li}^{37} \mathrm{Cl}$ and ${ }^{7} \mathrm{Li}^{35} \mathrm{Cl}$ and the isotope shift, i.e., the difference of $2 B$ between the isotopologues, is $6 \times 10^{-8}$ a.u., which is much smaller than $\omega_{\min }$. It is a bit surprising that the targeted control is largely successful, despite that the frequency spectrum is much less finer than the isotope shift. The mechanism of the isotope-selective rotational excitation might be explained as follows in terms of difference in the velocity of wave-packet motion between the isotopologues, leading to different quantum interference for different isotopologue. Assuming that the rotational wave packet is composed of two eigenstates and the transition energy is $\omega_{1}$ for one isotopologue and $\omega_{2}$ for the other, the time needed for the two wave packets to become out of phase by $\pi$ is estimated to be $\tau=\pi /\left|\omega_{1}-\omega_{2}\right|$ [13]. If the rotational wave packet for $\mathrm{LiCl}$ is composed of the lowest two eigenstates, $J=0$ and 1 , then $\tau$ is estimated to be $5.3 \times 10^{7}$ a.u., which is much longer than the present total time. Rotational wave packets created from higher quantum levels, however, can move faster and difference in phase between the two wave packets increases more quickly, resulting in a shorter $\tau$. For example, if the wave packet is created from the $J=0$ and 7 states, then $\tau$ is estimated to be $1.9 \times 10^{6}$ a.u., which is getting closer to the present total time. It has been shown in the control processes that high rotational levels are utilized to create rotational wave packets; in the OCT calculation with frequency cutoff (i) the rotational state is excited up to $J=4$, Figs. $2 \mathrm{c}$ and 2 d, and in the calculation with frequency cutoff (ii) the rotational state is excited up to $J=10$, Figs. $4 \mathrm{c}$ and $4 \mathrm{~d}$. Those results strongly suggest the control mechanism that rotational wave 
packets created from low and high quantum levels can achieve the high final yield to the target.

\subsection{2. $T=2560000$ a.u.}

First, we discuss the OCT result obtained with frequency cutoff (i). The final yield is 0,998 , the maximum field amplitude $1.019 \times 10^{-4}$ a.u., and the fluence $2.384 \times 10^{-3}$ a.u., Table II. The final yield is larger than that for $T=1280000$ a.u. and is nearly perfect. The maximum field amplitude and the field fluence are substantially smaller than those for $T$ $=1280000$ a.u. Figure 5a shows the optimal field as a function of $t$ and Fig. 5b its spectrum. The spectrum consists mainly of a frequency component around $\omega=0.003$ a.u., corresponding to the vibrational $v=1 \leftarrow 0$ transition energy, and of a component of smaller amplitude around $\omega=0.006$ a.u., corresponding to the first vibrational overtone. Shown in inset in Fig. 5b are the components of much smaller amplitudes in a low-frequency region; they accord with some rotational transitions. Calculating the final yield again with cutting out frequencies lower than $4 \times 10^{-4}$ a.u. from the optimal field resulted in a final yield less than 0.5 , indicating that the lower-frequency components play a significant role. Figure 6 shows temporal changes in the populations of vibrational and rotational states for the two isotopologues. At the beginning of the control pulse the populations of the vibrational $v=0$ state and the rotational $J=0$ state are 1.0 for both ${ }^{7} \mathrm{Li}^{37} \mathrm{Cl}$ and ${ }^{7} \mathrm{Li}^{35} \mathrm{Cl}$. The vibrational $v=1$ state is populated to some extent both in ${ }^{7} \mathrm{Li}^{37} \mathrm{Cl}$, Fig. 6 a, and ${ }^{7} \mathrm{Li}^{35} \mathrm{Cl}$, Fig. 6b, and almost no higher vibrational states are excited in contrast to the case for $T=$ 1280000 a.u. with frequency cutoff (i), Figs. $2 a$ and $2 b$. The rotational states, Figs. $6 c$ and 6d, exhibit similar behaviors to those for $T=1280000$ a.u. with frequency cutoff (i), Figs. $2 \mathrm{c}$ and $2 \mathrm{~d}$, although in the present case the amplitude of rapid oscillations is smaller. Finally, the populations of the target states, $J=1\left({ }^{7} \mathrm{Li}^{37} \mathrm{Cl}\right)$ and $J=0\left({ }^{7} \mathrm{Li}^{35} \mathrm{Cl}\right)$, reach nearly 1.0.

Next, the OCT result with frequency cutoff (ii) is presented. The final yield is 0,983 , the maximum field amplitude $3.285 \times 10^{-5}$ a.u., and the fluence $2.861 \times 10^{-4}$ a.u., Table II. The final yield is larger than that for $T=1280000$ a.u. and is nearly perfect. The maximum field amplitude and the field fluence are much smaller than those with frequency cutoff (i). Figure 7a shows the optimal field and Fig. 7b its spectrum. The 
optimal field changes very slowly and the spectrum is composed of frequency components lower than $1.5 \times 10^{-4}$ a.u. Shown in inset in Fig. $7 \mathrm{~b}$ is a close-up of the spectrum of frequencies less than $1.0 \times 10^{-4}$ a.u. and it is seen that the frequency component of the largest contribution lies around $7.0 \times 10^{-6}$ a.u. and that of the second largest contribution around $2.0 \times 10^{-5}$ a.u.; the former is close to the lowest $J=1 \leftarrow 0$ transition energy for $\mathrm{LiCl}$ equal to $2 B=6.45 \times 10^{-6}$ a.u. and the latter to the $J=3 \longleftarrow 2$ transition energy equal to $6 B=1.94 \times 10^{-5}$ a.u. In contrast to the spectrum for $T=1280000$ a.u. with frequency cutoff (ii), the component corresponding to the lowest rotational transition has the largest contribution in the spectrum for $T=2560000$ a.u. Figure 8 shows temporal changes in the populations of vibrational and rotational states for the two isotopologues. No change occurs in vibrational states by filtering, Figs. $8 \mathrm{a}$ and $8 \mathrm{~b}$, while rapid changes in rotational states are seen in Figs. 8c and 8d, but the changes are less rapid than those for $T=1280000$ a.u., Figs $4 \mathrm{c}$ and $4 \mathrm{~d}$, and the states are excited up to around $J=8$. The target states, $J=1\left({ }^{7} \mathrm{Li}^{37} \mathrm{Cl}\right)$ and $J=0\left({ }^{7} \mathrm{Li}^{35} \mathrm{Cl}\right)$, are finally achieved at a transition probability nearly 1.0.

The control mechanism when using frequency cutoff (ii) can be explained in the same way as the previous subsection for $T=1280000$ a.u., because the minimum frequency that can be represented by this pulse, $2.5 \times 10^{-6}$ a.u. is still much larger than the isotope shift in the rotational transition energy, $6 \times 10^{-8}$ a.u., whereas the obtained final yield is nearly perfect. The velocity of the wave packet created from high rotational eigenstates makes distinct difference between the isotopologues in a shorter time than that created from lower eigenstates, leading, in the last stage of the pulse, to large difference in the phase of wave packet; because of the difference in phase, the wave packet for different isotopologue experiences different quantum interference in the last stage, achieving finally $J=1$ for ${ }^{7} \mathrm{Li}^{37} \mathrm{Cl}$ and $J=0$ for ${ }^{7} \mathrm{Li}^{35} \mathrm{Cl}$

\subsection{Vibrational-rotational excitation}

In this subsection the OCT results for the isotope-selective vibrational-rotational

excitation, ${ }^{7} \mathrm{Li}{ }^{37} \mathrm{Cl}(v=0, J=0) ;{ }^{7} \mathrm{Li}^{35} \mathrm{Cl}(v=0, J=0) \rightarrow{ }^{7} \mathrm{Li}^{37} \mathrm{Cl}(v=1, J=1) ;{ }^{7} \mathrm{Li}^{35} \mathrm{Cl}(v$ $=0, J=0)$, are presented and some detailed discussion is given for each total time. In the present OCT calculations frequencies lower than $1.5 \times 10^{-3}$ a.u. are cut out for $T=640000$ 
a.u. and so are those lower than $1.0 \times 10^{-4}$ a.u. for $T=1280000$ a.u., i.e., the frequencies corresponding to transitions between lower rotational levels are removed from the control field. It has been found that this effectively smooths the complex temporal change in rotational-level populations and makes the control mechanism quite simple, while maintaining the final yields at high levels.

\subsection{1. $T=640000$ a.u.}

As summarized in Table II, the final yield obtained for $T=640000$ is 0.765 ; the maximum field amplitude and fluence are $2.500 \times 10^{-3}$ a.u. and $9.730 \times 10^{-2}$ a.u., respectively. Figure 9a shows the optimized field and Fig. $9 \mathrm{~b}$ its spectrum; the amplitude of the field is very large around $t=300000$ a.u.; in the spectrum some overtones as well as the fundamental appear and the first overtone plays a predominant role with the others having secondary importance. This strongly suggests that the control for the present total time needs to make use of overtone transitions to create wave packets that can move quickly, thus achieving the highest final yield possible. The large amplitude of the field around $t=300000$ a.u. can thus be explained by the overtone transitions that need very strong fields because overtone transition moments are generally very small. Figure 10 shows temporal changes in the populations of vibrational and rotational states for the two isotopologues, caused by the obtained field. The populations of the $v=1$ and $J=1$ states for ${ }^{7} \mathrm{Li}^{37} \mathrm{Cl}$, Figs. 10a and 10c, reach about 0.85 , while those of the other states decrease to nearly zero, at the end of the pulse. The populations of the vibrational and rotational states for ${ }^{7} \mathrm{Li}^{35} \mathrm{Cl}$, Figs. $10 \mathrm{~b}$ and $10 \mathrm{~d}$, exhibit significant changes in the middle of the pulse, but they almost return back to the initial situation at the end where only the ground states are populated, although some vibrational and rotational excited states are slightly populated. The state populations exhibit large oscillations around $t=300000$ a.u., which is due to the large field amplitude around this time, Fig. 9a.

The vibrational-rotational transition energies from $(v=0, J=0)$ to $(v=1, J=1)$ are 0.002963 a.u. and 0.002976 a.u., respectively, for ${ }^{7} \mathrm{Li}^{37} \mathrm{Cl}$ and ${ }^{7} \mathrm{Li}^{35} \mathrm{Cl}$, Table III, and the isotope shift, i.e., the difference in transition energy between the isotopologues, is $1.4 \mathrm{x}$ $10^{-5}$ a.u. The minimum frequency for $T=640000$ a.u. is $\omega_{\min }=2 \pi / T=9.8 \times 10^{-6}$ a.u., which is comparable with the isotope shift, and hence the control of the present isotope- 
selective vibrational-rotational transition is achieved rather successfully. Note that the isotope shift in the pure rotational transition $J=1 \leftarrow 0$ is $6 \times 10^{-8}$ a.u., which is much smaller than that in the present vibrational-rotational transition, and no control of pure rotational transition was obtained for the same total time.

\subsection{2. $T=1280000$ a.u.}

As shown in Table II, the final yield for $T=1280000$ a.u. is 0.988 , which is nearly perfect and substantially higher than that for $T=640000$ a.u; the maximum field amplitude and fluence are $7.289 \times 10^{-5}$ a.u. and $1.206 \times 10^{-3}$ a.u., respectively, which are much smaller than those for $T=640000$ a.u. Figure 11 shows (a) the optimal field as a function of $t$ and (b) its spectrum. The spectrum shows that only the fundamental frequency for the vibrational transition plays a role. The inset in Fig. 11b shows a closeup of the spectrum around $\omega=0.003$ a.u.; it is seen that the peak is at 0.00296 a.u. The vibrational-rotational transition energy from $(v=0, J=0)$ to $(v=1, J=1)$ is calculated to be 0.002963 a.u. for ${ }^{7} \mathrm{Li}^{37} \mathrm{Cl}$ and 0.002976 a.u. for ${ }^{7} \mathrm{Li}^{35} \mathrm{Cl}$, Table III. The peak in the spectrum exactly matches the transition energy for ${ }^{7} \mathrm{Li}^{37} \mathrm{Cl}$. Figure 12 shows temporal changes in the populations of vibrational and rotational states for the two isotopologues. For ${ }^{7} \mathrm{Li}^{37} \mathrm{Cl}$ vibrational and rotational states exhibit almost the same change and the populations of $v=1$ and $J=1$ reach nearly 1.0 with those of the other states decreasing to zero at the end of the pulse, Figs. 12a and 12c. On the other hand, vibrational and rotational states for ${ }^{7} \mathrm{Li}^{35} \mathrm{Cl}$ show almost no change, Figs. $12 \mathrm{~b}$ and $12 \mathrm{~d}$.

$\omega_{\min }$ for $T=1280000$ a.u. is $4.9 \times 10^{-6}$ a.u., which is less than half the isotope shift in the vibrational-rotational transition energies, $1.4 \times 10^{-5}$ a.u. Thus the control mechanism here is explained by what is called the conventional laser isotope separation; the separation relies only on the applied laser frequency and does not use difference in quantum interference between wave packets moving in different velocity, which are important in the isotope-selective pure rotational excitation, as discussed in the previous section.

\subsection{3. $T=2560000$ a.u.}


The final yield for $T=2560000$ a.u. is 0.999 , which is nearly perfect and slightly higher than that for $T=1280000 \mathrm{a}$.u; the maximum field amplitude and fluence are 4.860 x $10^{-5}$ a.u. and $8.151 \times 10^{-4}$ a.u., which are slightly smaller than those for $T=1280000$ a.u., Table II. Figure 13 shows (a) the optimal field as a function of $t$ and (b) its spectrum. The spectrum includes only the fundamental frequency and no overtone appears. A closeup of the spectrum around $\omega=0.003$ a.u., Fig. $13 \mathrm{~b}$, shows again that the peak at $\omega=$ 0.00296 a.u. exactly matches the vibrational-rotational transition energy from $(v=0, J=$ $0)$ to $(v=1, J=1)$ for ${ }^{7} \mathrm{Li}^{37} \mathrm{Cl}, 0.002963$ a.u. Figure 14 shows the temporal changes in the populations of vibrational and rotational states for the two isotopologues. Those state changes are very similar to those for $T=1280000$ a.u., Fig. 12, although in the present case much less state excitation occurs in ${ }^{7} \mathrm{Li}^{35} \mathrm{Cl}$, Figs. $14 \mathrm{~b}$ and $14 \mathrm{~d}$, compared with Figs. $12 \mathrm{~b}$ and $12 \mathrm{~d}$. It is thus concluded that the control mechanism for $T=2560000$ a.u. is the same as that for $T=1280000$ a.u., where only the laser frequency is the key factor for isotope-selective excitation.

\section{Conclusion}

In this work we have carried out OCT calculations to obtain the electric fields that effectively steer isotope-selective transitions for the fifty-fifty mixture of diatomic isotopologues, ${ }^{7} \mathrm{Li}^{37} \mathrm{Cl}$ and ${ }^{7} \mathrm{Li}^{35} \mathrm{Cl}$. The two-dimensional time-dependent wave packet propagation was calculated with both the radial and angular motions of the diatomic molecule being treated quantum mechanically. We investigated quantum controls of the isotope-selective pure rotational and vibrational-rotational excitations of the diatomic molecules. In all the controls the initial states were set to the situation that both ${ }^{7} \mathrm{Li}^{37} \mathrm{Cl}$ and ${ }^{7} \mathrm{Li}^{35} \mathrm{Cl}$ are in the $v=0$ and $J=0$ states. The target in the pure rotational excitation was ${ }^{7} \mathrm{Li}^{37} \mathrm{Cl}(v=0, J=1)$ and ${ }^{7} \mathrm{Li}^{35} \mathrm{Cl}(v=0, J=0)$ and that in the vibrational-rotational excitation was ${ }^{7} \mathrm{Li}^{37} \mathrm{Cl}(v=1, J=1)$ and ${ }^{7} \mathrm{Li}^{35} \mathrm{Cl}(v=0, J=0)$. Two total times 128000 and 2560000 a.u. (31.0 and 61.9 ps) were examined for the pure rotational excitation and three total times, 640000, 128000, and 2560000 a.u. $(15.5,31.0$, and $61.9 \mathrm{ps})$ for the vibrational-rotational excitation. The obtained final yields for the pure rotational excitation were just over 0.9 for $T=1280000$ a.u. and nearly 1.0 for $T=2560000$ a.u. and those for the vibrational-rotational excitation were just less than 0.8 for $T=640000$ a.u. 
and nearly 1.0 for $T=1280000$ and 2560000 a.u. It was found that the isotope-selective pure rotational excitation can be realized by two different control fields: One consists of frequency components corresponding to both the vibrational and rotational transitions and the other consists of components corresponding to only the rotational transition. The control fields for the vibrational-rotational excitations were found to include components corresponding to the vibrational transition accompanied by the rotational transition. We have thus theoretically succeeded in controlling the isotope-selective transitions of diatomic molecules using the method including both radial and angular motions quantum mechanically. The future direction might be aiming at the control of a mixed ensemble of diatomic molecules having a distribution over many quantum states characterized by its temperature, which will perhaps lead to a breakthrough in the conventional industrial technology such as isotope separation of nuclear fuel waste.

\section{Acknowledgements}

The computations were performed using the supercomputers at Center for Computational Science \& e-Systems, JAEA, Tokai, Ibaraki, Japan and also at Research Center for Computational Science, Okazaki, Aichi, Japan. This work was supported by JSPS KAKENHI Grant No. 15H02345. 


\section{References}

[1] S. A. Rice and M. Zhao, Optical Control of Molecular Dynamics, Wiley, New York, 2000.

[2] V. S. Letokhov, Laser Control of Atoms and Molecules, Oxford University Press, New York, 2007.

[3] D. D'alessandro, Introduction to Quantum Control and Dynamics, Chapman \& Hall, 2007.

[4] M. Shapiro and P. Brumer, Quantum Control of Molecular Processes, Wiley-VCH, Weinheim, 2012.

[5] B. Li, G. Turinici, V. Ramakrishna, H. Rabitz, J. Phys. Chem. B 106 (2002) 8125.

[6] G. Turinici, V. Ramakrishna, B. Li, H. Rabitz, J. Phys. A 37 (2004) 273.

[7] G. Turinici, H. Rabitz, Phys. Rev. A 70 (2004) 063412.

[8] A. Lindinger, C. Lupulescu, M. Plewicki, F. Vetter, A. Merli, S. M. Weber, L. Wöste, Phys. Rev. Lett. 93 (2004) 033001.

[9] F. Vetter, M. Plewicki, A. Lindinger, A. Merli, S. M. Weber, L. Wöste, Phys. Chem. Chem. Phys. 7, 1151 (2005).

[10] M. Roth, L. Guyon, J. Roslund, V. Boutou, F. Courvoisier, J.-P. Wolf, H. Rabitz, Phys. Rev. Lett. 102 (2009) 253001.

[11] J. Roslund, M. Roth, L. Guyon, V. Boutou, F. Courvoisier, J.-P. Wolf, H. Rabitz, J. Chem. Phys. 134 (2011) 034511.

[12] Y. Kurosaki, K. Yokoyama, A. Yokoyama, J. Mol. Struct. THEOCHEM 913 (2009) 38.

[13] Y. Kurosaki, K. Yokoyama, A. Yokoyama, J. Chem. Phys. 131 (2009) 144305.

[14] Y. Kurosaki, K. Yokoyama, A. Yokoyama, Comput. Theoret. Chem. 963 (2011) 245.

[15] Y. Kurosaki, A. Ichihara, K. Yokoyama, J. Chem. Phys. 135 (2011) 054103.

[16] S. Shi, H. Rabitz, J. Chem. Phys. 92 (1990) 364.

[17] W. Zhu, J. Botina, H. Rabitz, J. Chem. Phys. 108 (1998) 1953.

[18] K. Sundermann, R. de Vivie-Riedle, J. Chem. Phys. 110 (1999) 1896.

[19] K. Yokoyama, L. Matsuoka, T. Kasajima, M. Tsubouchi, A. Yokoyama in Advances in Intense Laser Science and Photonics ed by J. Lee, Publishing House for Science and Technology, Hanoi, 2010, p. 113. 
[20] L. Matsuoka, A. Ichihara, M. Hashimoto, K. Yokoyama, Proc. Int. Conf. on Toward and Over the Fukushima Daiichi Accident (GLOBAL 2011), 2011, 5017532

[21] A. Ichihara, L. Matsuoka, Y. Kurosaki, K. Yokoyama, Proc. $12^{\text {th }}$ Asia Pacific Physics Conference, Chiba, 2013, JPS Conf. Proc. 1 (2014) 013093.

[22] A. Ichihara, L. Matsuoka, Y. Kurosaki, K. Yokoyama, Chinese J. Phys. 51 (2013) 1230.

[23] A. Ichihara, L. Matsuoka, Y. Kurosaki, K. Yokoyama, Opt. Rev. 22 (2015) 153.

[24] Y. Maday, G. Turinici, J. Chem. Phys. 118 (2003) 8191.

[25] Y. Kurosaki, K. Yokoyama, J. Chem. Phys. 137 (2012) 064305.

[26] C. C. Marston, G. G. Balint-Kurti, J. Chem. Phys. 91 (1989) 3571.

[27] M. D. Feit, J. A. Fleck, Jr., A. Steiger, J. Comput. Phys. 47 (1982) 412.

[28] M. D. Feit, J. A. Fleck, Jr., J. Chem. Phys. 78 (1983) 301.

[29] M. D. Feit, J. A. Fleck, Jr., J. Chem. Phys. 80 (1984) 2578.

[30] W. H. Press, B. P. Flannery, S. A. Teukolsky, and . T. Vetterling, Numerical Recipes, Cambridge University Press, Cambridge, MA, 1986.

[31] J. C. Light, I. P. Hamilton, V. J. Lill, J. Chem. Phys. 82 (1985) 1400.

[32] S. Mahapatra, N. Sathyamurthy, J. Chem. Soc., Faraday Trans. 93 (1997) 773. 


\section{Table 1}

Grid and time parameters used in the present OCT calculations

\begin{tabular}{lccc}
\hline \multicolumn{1}{c}{ Parameter } & Value \\
\hline Range of the Li-Cl bond length (bohr) & $2.2677-5.6692$ \\
Number of radial grid points & 32 & \\
Number of angular grid points & 16 & \\
Total time (a.u.) & 640000 & 1280000 & 256000 \\
Number of time steps & 131072 & 262144 & 524288 \\
\hline
\end{tabular}




\section{Table 2}

Properties of the resultant optimal electric fields

\begin{tabular}{|c|c|c|c|c|c|c|}
\hline$T / \mathrm{au}$ & $\alpha_{0}$ & $\delta$ & $\eta$ & $\begin{array}{l}\text { Max. field } \\
\text { amp. / a.u. }\end{array}$ & $\begin{array}{l}\text { Fluence / } \\
\text { a.u. }\end{array}$ & Yield \\
\hline \multicolumn{7}{|c|}{$\begin{array}{l}\text { Rotational excitation: }{ }^{7} \mathrm{Li}^{37} \mathrm{Cl}(v=0, J=0) ;{ }^{7} \mathrm{Li}^{35} \mathrm{Cl}(v=0, J=0) \rightarrow{ }^{7} \mathrm{Li}^{37} \mathrm{Cl}(v=0, J=1) \text {; } \\
{ }^{7} \mathrm{Li}^{35} \mathrm{Cl}(v=0, J=0)\end{array}$} \\
\hline $1,280,000(\sim 31.0 \mathrm{ps})^{\mathrm{a}}$ & 10.0 & 0.01 & 0.01 & $3.014 \times 10^{-3}$ & $2.606 \times 10^{-1}$ & 0.903 \\
\hline $1,280,000(\sim 31.0 \mathrm{ps})^{\mathrm{b}}$ & 0.5 & 0.01 & 0.01 & $6.552 \times 10^{-4}$ & $6.201 \times 10^{-2}$ & 0.921 \\
\hline $2,560,000(\sim 61.9 \mathrm{ps})^{\mathrm{a}}$ & 10.0 & 0.01 & 0.01 & $1.019 \times 10^{-4}$ & $2.384 \times 10^{-3}$ & 0.998 \\
\hline $2,560,000(\sim 61.9 \mathrm{ps})^{\mathrm{b}}$ & 100.0 & 0.01 & 0.01 & $3.285 \times 10^{-5}$ & $2.861 \times 10^{-4}$ & 0.983 \\
\hline \multicolumn{7}{|c|}{$\begin{array}{l}\text { Vibrational-rotational excitation: }{ }^{7} \mathrm{Li}^{37} \mathrm{Cl}(v=0, J=0) ;{ }^{7} \mathrm{Li}^{35} \mathrm{Cl}(v=0, J=0) \rightarrow{ }^{7} \mathrm{Li}^{37} \mathrm{Cl}(v \\
=1, J=1) ;{ }^{7} \mathrm{Li}^{35} \mathrm{Cl}(v=0, J=0)\end{array}$} \\
\hline $640,000(\sim 15.5 \mathrm{ps})$ & 1.0 & 0.05 & 0.05 & $2.500 \times 10^{-3}$ & $9.730 \times 10^{-2}$ & 0.765 \\
\hline $1,280,000(\sim 31.0 \mathrm{ps})$ & 50.0 & 0.01 & 0.01 & $7.289 \times 10^{-5}$ & $1.206 \times 10^{-3}$ & 0.988 \\
\hline $2,560,000(\sim 61.9 \mathrm{ps})$ & 50.0 & 0.01 & 0.01 & $4.860 \times 10^{-5}$ & $8.151 \times 10^{-4}$ & 0.999 \\
\hline
\end{tabular}

${ }^{\mathrm{a}}$ Using frequency cutoff (i).

bUsing frequency cutoff (ii). 
Table 3

Rovibrational state energies for ${ }^{7} \mathrm{Li}^{37} \mathrm{Cl}$ and ${ }^{7} \mathrm{Li}^{35} \mathrm{Cl}$ (a.u.).

\begin{tabular}{ccccccc}
\hline \multicolumn{5}{c}{${ }^{7} \mathrm{Li}^{37} \mathrm{Cl}$} & \multicolumn{3}{c}{${ }^{7} \mathrm{Li}^{35} \mathrm{Cl}$} \\
\hline & $J$ & 2 & 0 & 1 & 2 \\
\hline$v$ & 0 & 1 & -0.154194 & -0.154206 & -0.154200 & -0.154187 \\
\hline 0 & -0.154213 & -0.154207 & -0.151237 & -0.151230 & -0.151217 \\
1 & -0.151257 & -0.151250 & -0.151237 & -0.151237 \\
2 & -0.148341 & -0.148335 & -0.148322 & -0.148309 & -0.148302 & -0.148289 \\
\hline
\end{tabular}




\section{Figure captions}

Fig. 1. (a) The optimal control field $\varepsilon(t)$ for the isotope-selective rotational excitation with frequency cutoff (i) for $T=1280000$ a.u.; (b) the spectrum of $\varepsilon(t)$.

Fig. 2. Temporal changes in state populations for the isotope-selective rotational excitation with frequency cutoff (i) for $T=1280000$ a.u.: (a) vibrational states for ${ }^{7} \mathrm{Li}^{37} \mathrm{Cl}$; (b) vibrational states for ${ }^{7} \mathrm{Li}^{35} \mathrm{Cl}$; (c) rotational states for ${ }^{7} \mathrm{Li}^{37} \mathrm{Cl}$; (d) rotational states for ${ }^{7} \mathrm{Li}^{35} \mathrm{Cl}$

Fig. 3. (a) The optimal control field $g(t)$ for the isotope-selective rotational excitation with frequency cutoff (ii) for $T=1280000$ a.u.; (b) the spectrum of $\varepsilon(t)$.

Fig. 4. Temporal changes in state populations for the isotope-selective rotational excitation with frequency cutoff (ii) for $T=1280000$ a.u.: (a) vibrational states for ${ }^{7} \mathrm{Li}^{37} \mathrm{Cl}$; (b) vibrational states for ${ }^{7} \mathrm{Li}^{35} \mathrm{Cl}$; (c) rotational states for ${ }^{7} \mathrm{Li}^{37} \mathrm{Cl}$; (d) rotational states for ${ }^{7} \mathrm{Li}^{35} \mathrm{Cl}$

Fig. 5. (a) The optimal control field $\varepsilon(t)$ for the isotope-selective rotational excitation with frequency cutoff (i) for $T=2560000$ a.u.; (b) the spectrum of $\varepsilon(t)$.

Fig. 6. Temporal changes in state populations for the isotope-selective rotational excitation with frequency cutoff (i) for $T=2560000$ a.u.: (a) vibrational states for ${ }^{7} \mathrm{Li}^{37} \mathrm{Cl}$; (b) vibrational states for ${ }^{7} \mathrm{Li}^{35} \mathrm{Cl}$; (c) rotational states for ${ }^{7} \mathrm{Li}^{37} \mathrm{Cl}$; (d) rotational states for ${ }^{7} \mathrm{Li}^{35} \mathrm{Cl}$

Fig. 7. (a) The optimal control field $\varepsilon(t)$ for the isotope-selective rotational excitation with frequency cutoff (ii) for $T=2560000$ a.u., obtained by filtering out the frequencies larger than 0.001 a.u.; (b) the spectrum of $\varepsilon(t)$.

Fig. 8. Temporal changes in state populations for the isotope-selective rotational excitation with frequency cutoff (ii) for $T=2560000$ a.u., obtained by filtering out the frequencies larger than 0.001 a.u.: (a) vibrational states for ${ }^{7} \mathrm{Li}^{37} \mathrm{Cl}$; (b) vibrational states for ${ }^{7} \mathrm{Li}^{35} \mathrm{Cl}$; (c) rotational states for ${ }^{7} \mathrm{Li}^{37} \mathrm{Cl}$; (d) rotational states for ${ }^{7} \mathrm{Li}^{35} \mathrm{Cl}$.

Fig. 9. (a) The optimal control field $\varepsilon(t)$ for the isotope-selective vibrational-rotational excitation for $T=640000$ a.u.; (b) the spectrum of $\varepsilon(t)$.

Fig. 10. Temporal changes in state populations for the isotope-selective vibrationalrotational excitation for $T=640000$ a.u.: (a) vibrational states for ${ }^{7} \mathrm{Li}^{37} \mathrm{Cl}$; (b) 
vibrational states for ${ }^{7} \mathrm{Li}^{35} \mathrm{Cl}$; (c) rotational states for ${ }^{7} \mathrm{Li}^{37} \mathrm{Cl}$; (d) rotational states for ${ }^{7} \mathrm{Li}^{35} \mathrm{Cl}$

Fig. 11. (a) The optimal control field $\varepsilon(t)$ for the isotope-selective vibrational-rotational excitation for $T=1280000$ a.u.; (b) the spectrum of $\varepsilon(t)$.

Fig. 12. Temporal changes in state populations for the isotope-selective vibrationalrotational excitation for $T=1280000$ a.u.: (a) vibrational states for ${ }^{7} \mathrm{Li}^{37} \mathrm{Cl}$; (b) vibrational states for ${ }^{7} \mathrm{Li}^{35} \mathrm{Cl}$; (c) rotational states for ${ }^{7} \mathrm{Li}^{37} \mathrm{Cl}$; (d) rotational states for ${ }^{7} \mathrm{Li}^{35} \mathrm{Cl}$

Fig. 13. (a) The optimal control field $\varepsilon(t)$ for the isotope-selective vibrational-rotational excitation for $T=2560000$ a.u.; (b) the spectrum of $\varepsilon(t)$.

Fig. 14. Temporal changes in state populations for the isotope-selective vibrationalrotational excitation for $T=2560000$ a.u.: (a) vibrational states for ${ }^{7} \mathrm{Li}^{37} \mathrm{Cl}$; (b) vibrational states for ${ }^{7} \mathrm{Li}^{35} \mathrm{Cl}$; (c) rotational states for ${ }^{7} \mathrm{Li}^{37} \mathrm{Cl}$; (d) rotational states for ${ }^{7} \mathrm{Li}^{35} \mathrm{Cl}$ 

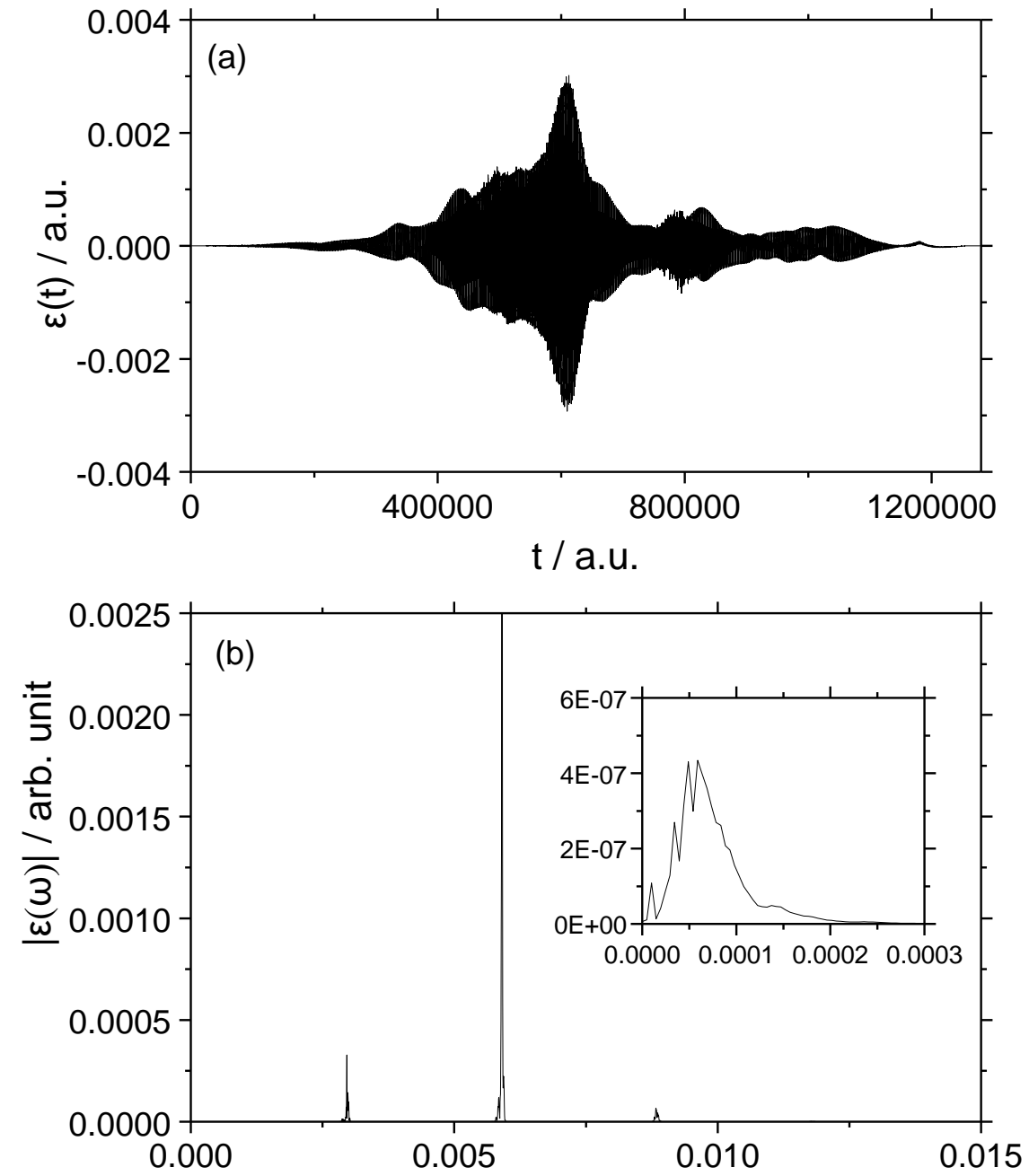

w/a.u.

Fig. 1 

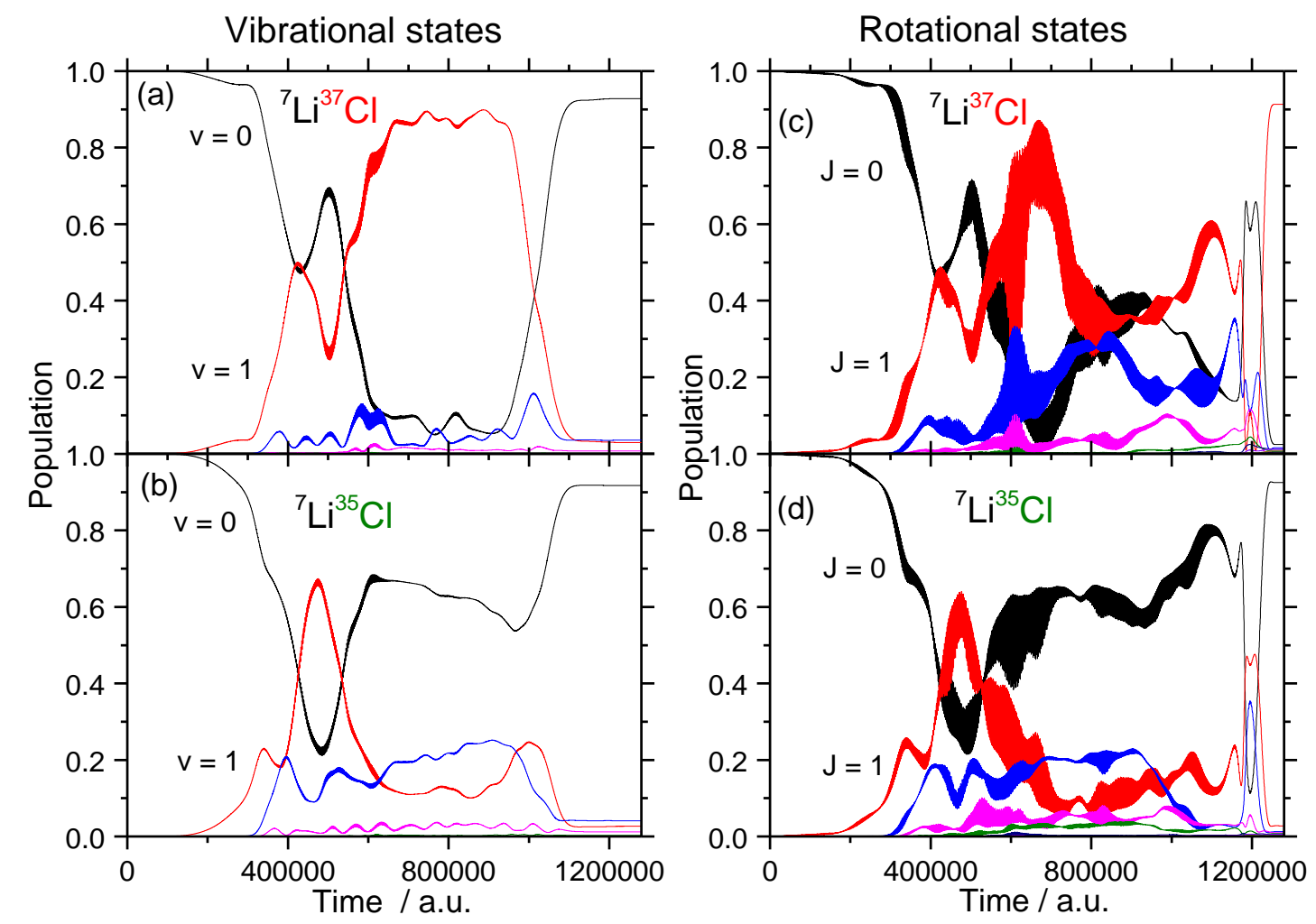

Fig. 2 

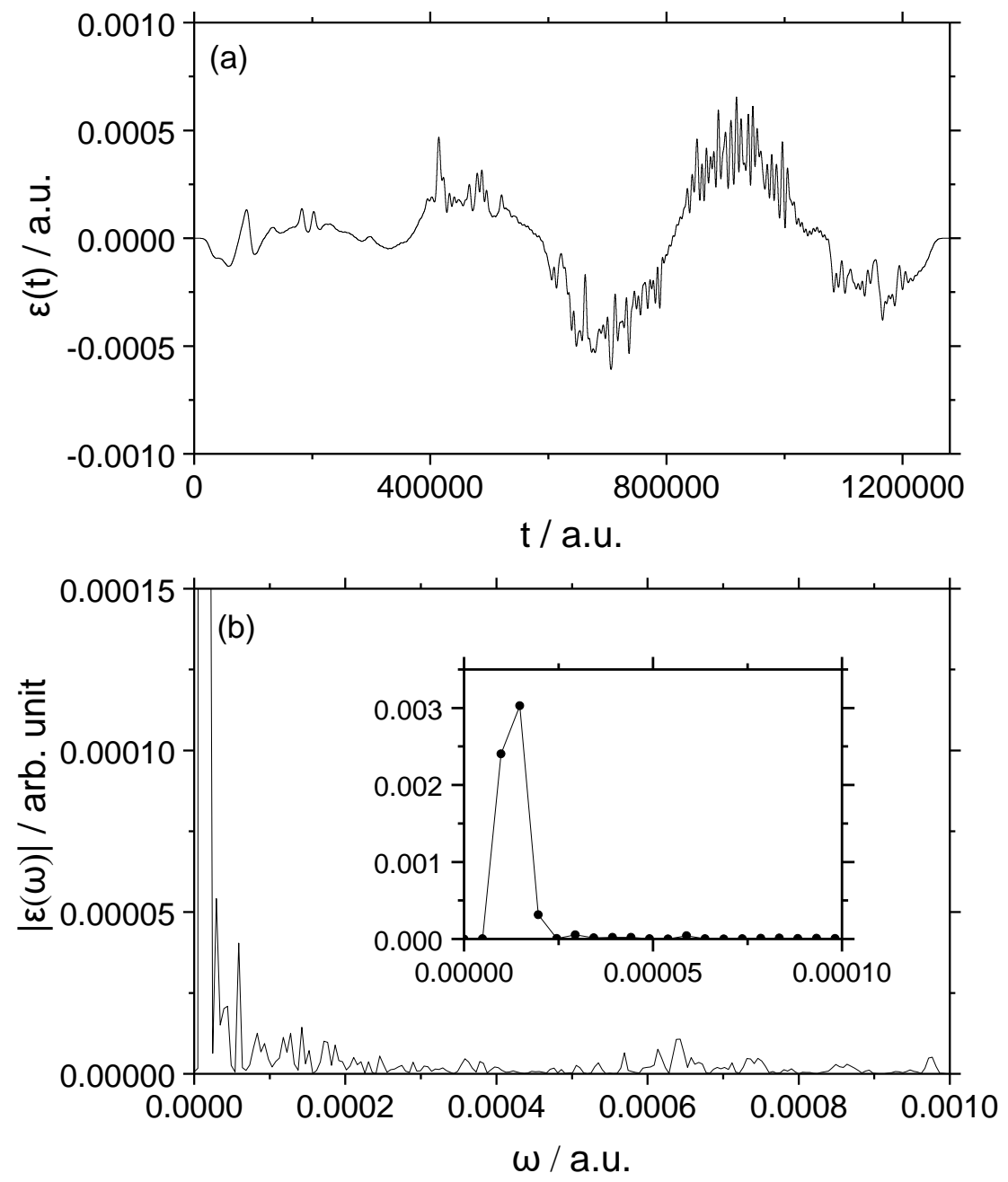

Fig. 3 

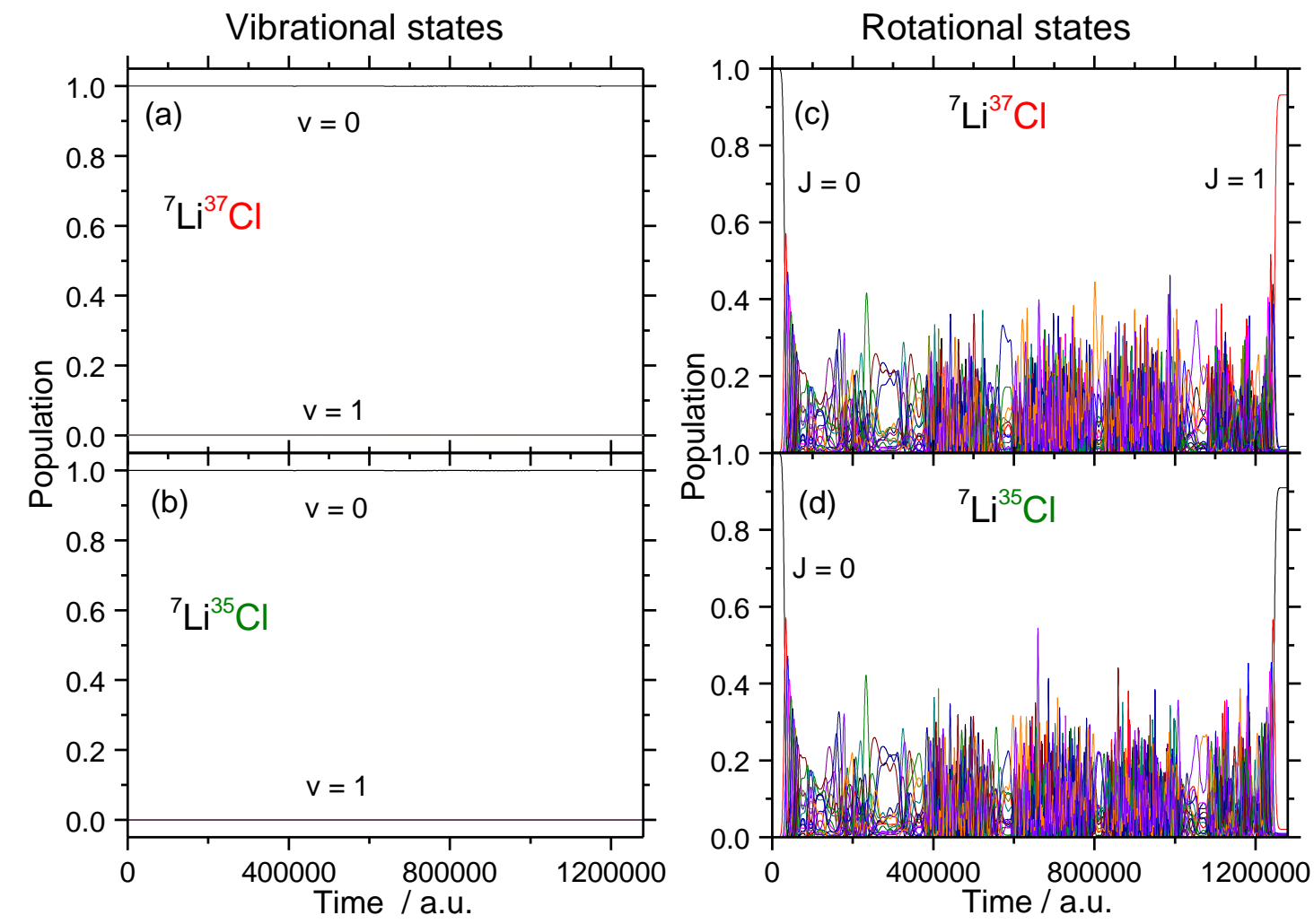

Fig. 4 

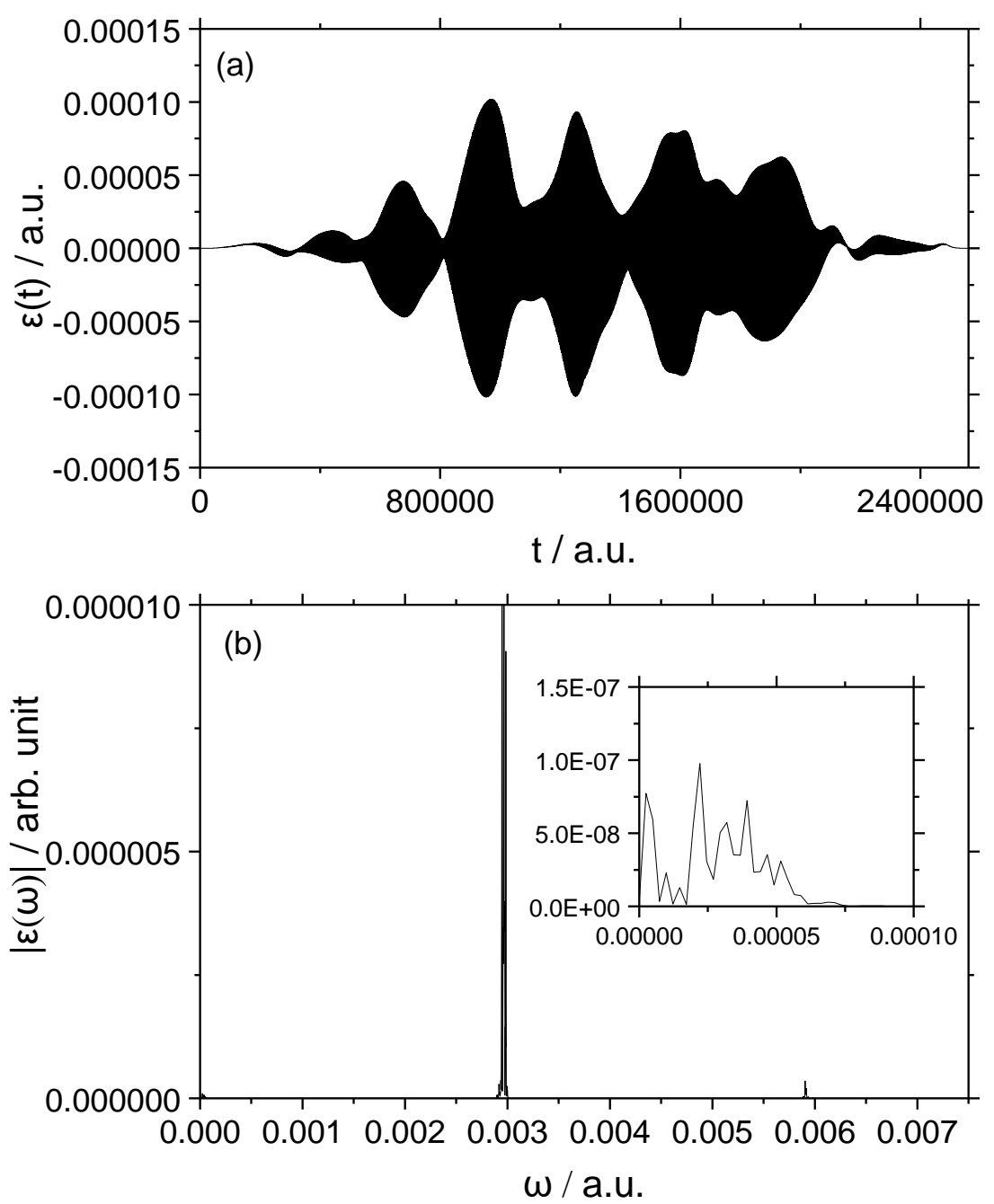

Fig.5 

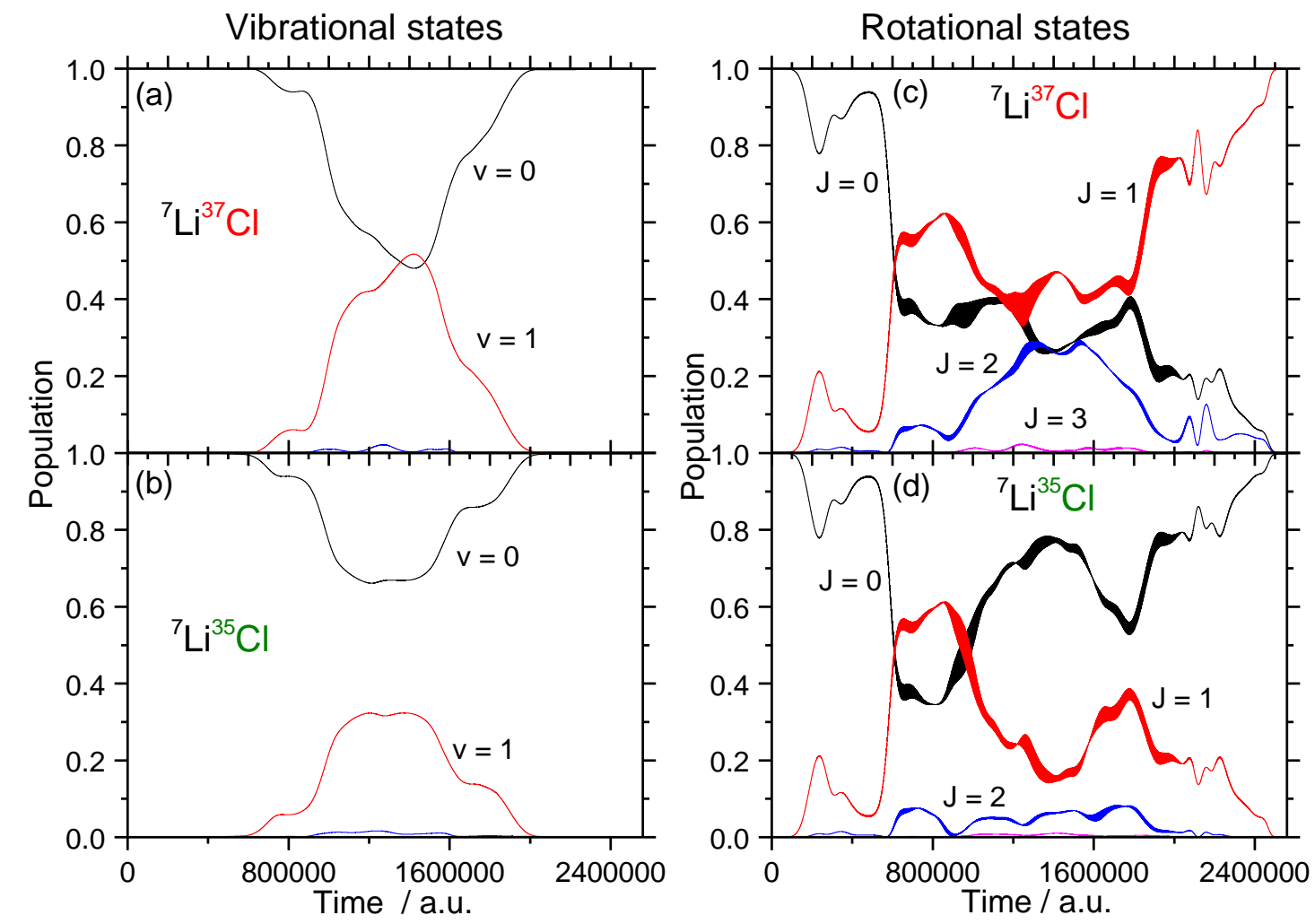

Fig. 6 

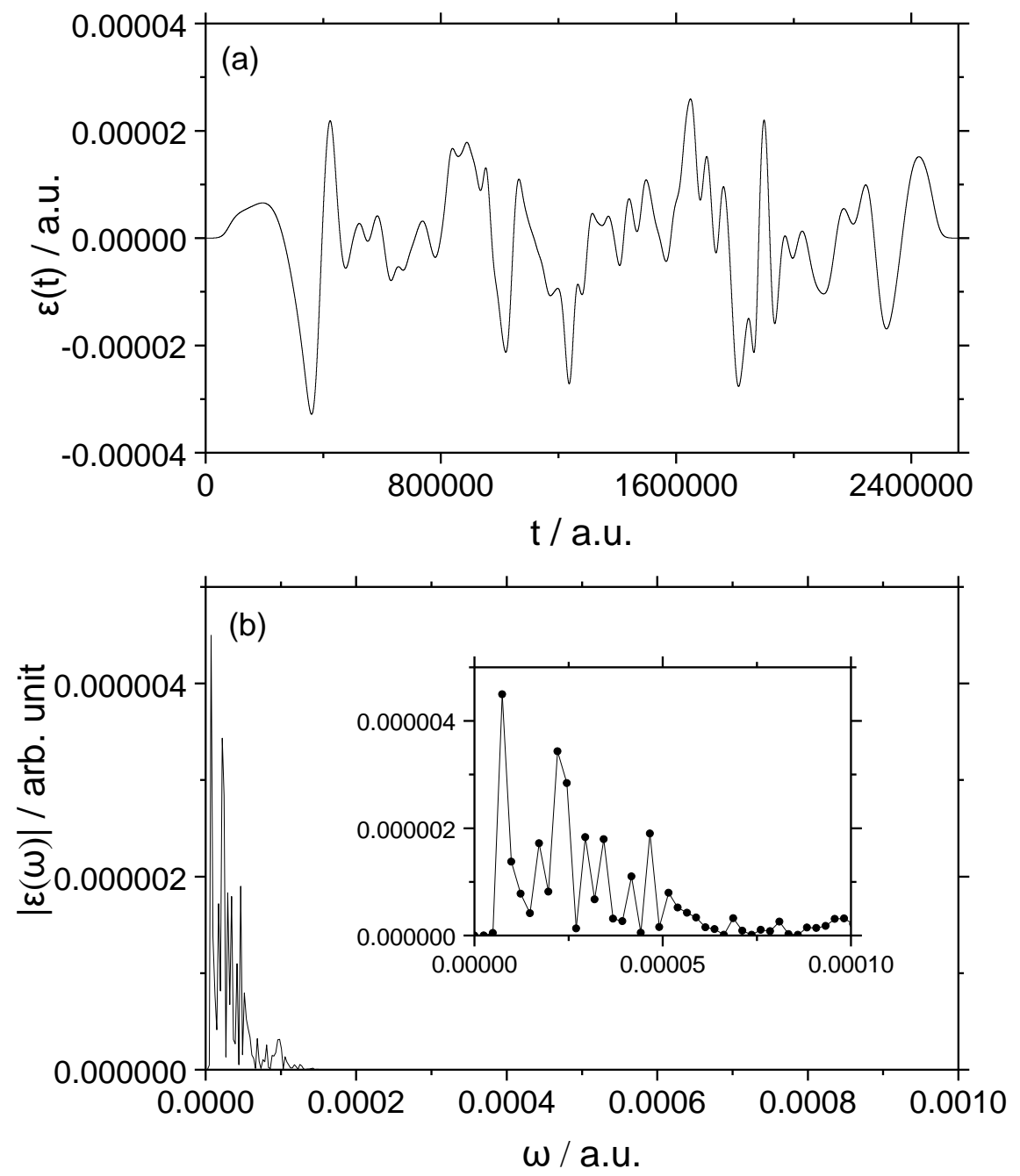

Fig. 7 

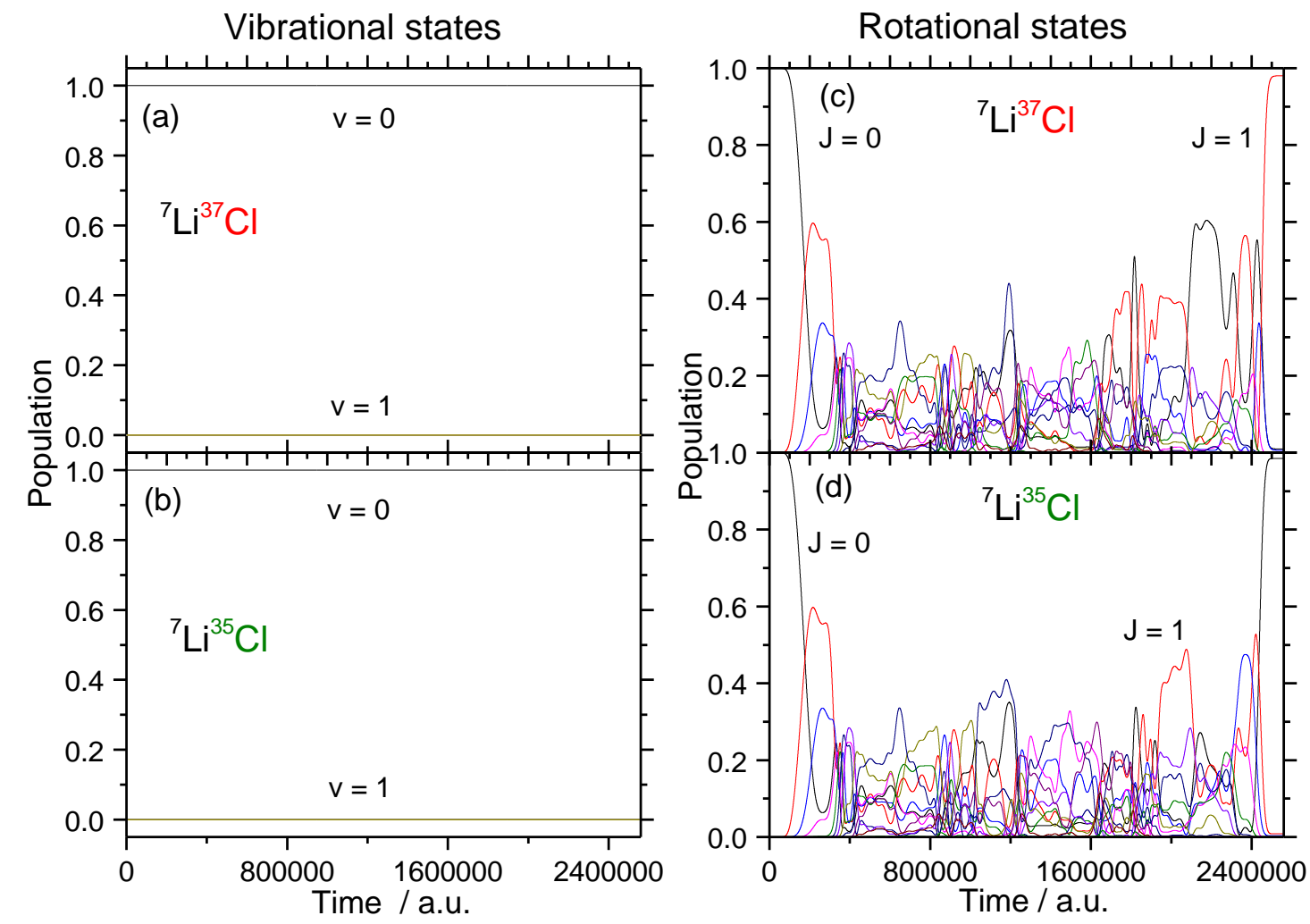

Fig. 8 

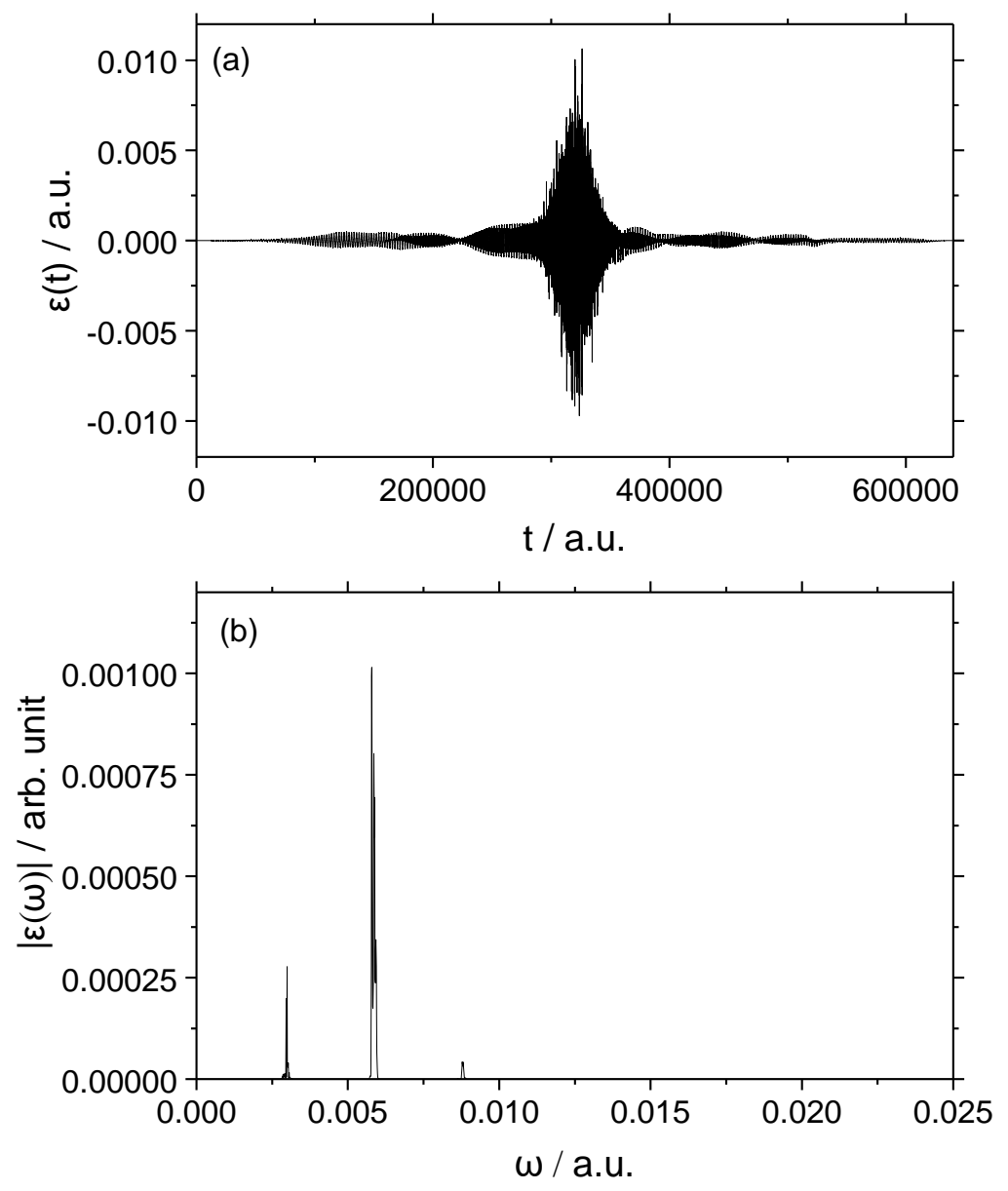

Fig. 9 
Vibrational states

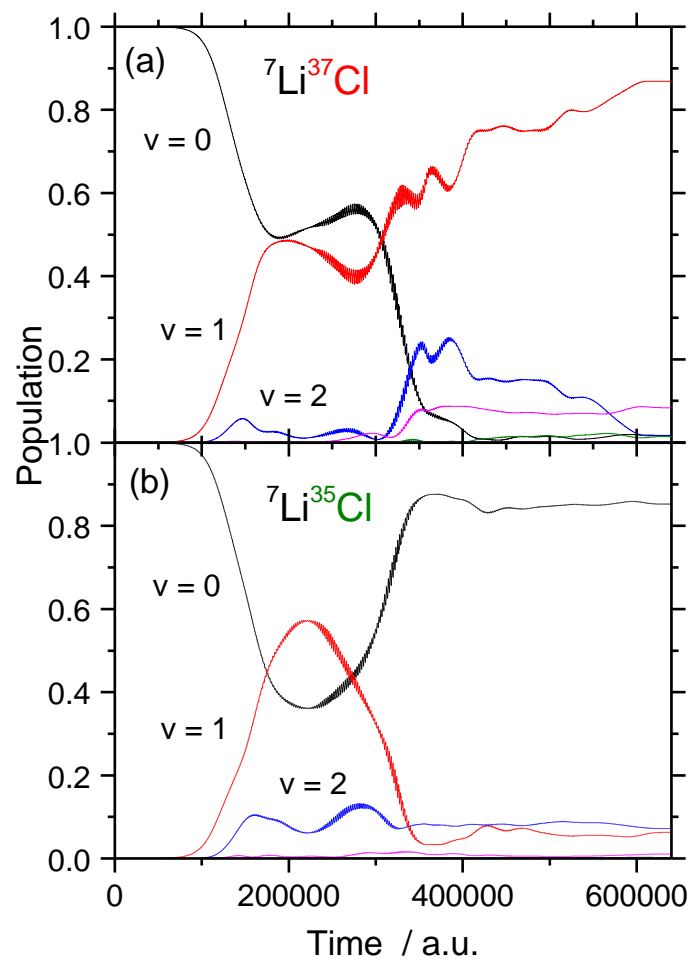

Rotational states

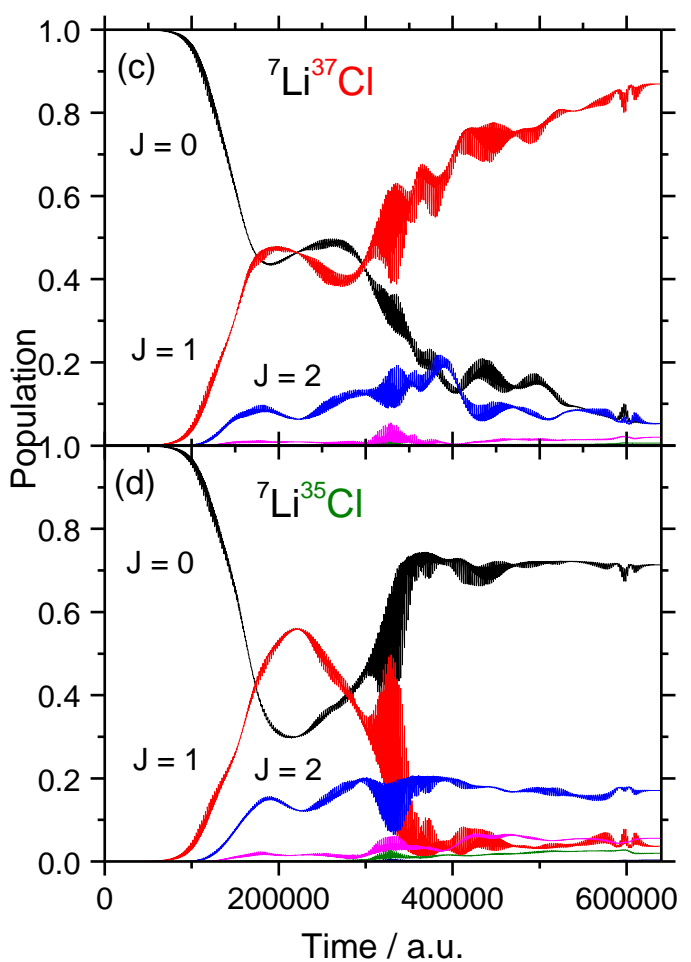

Fig. 10 

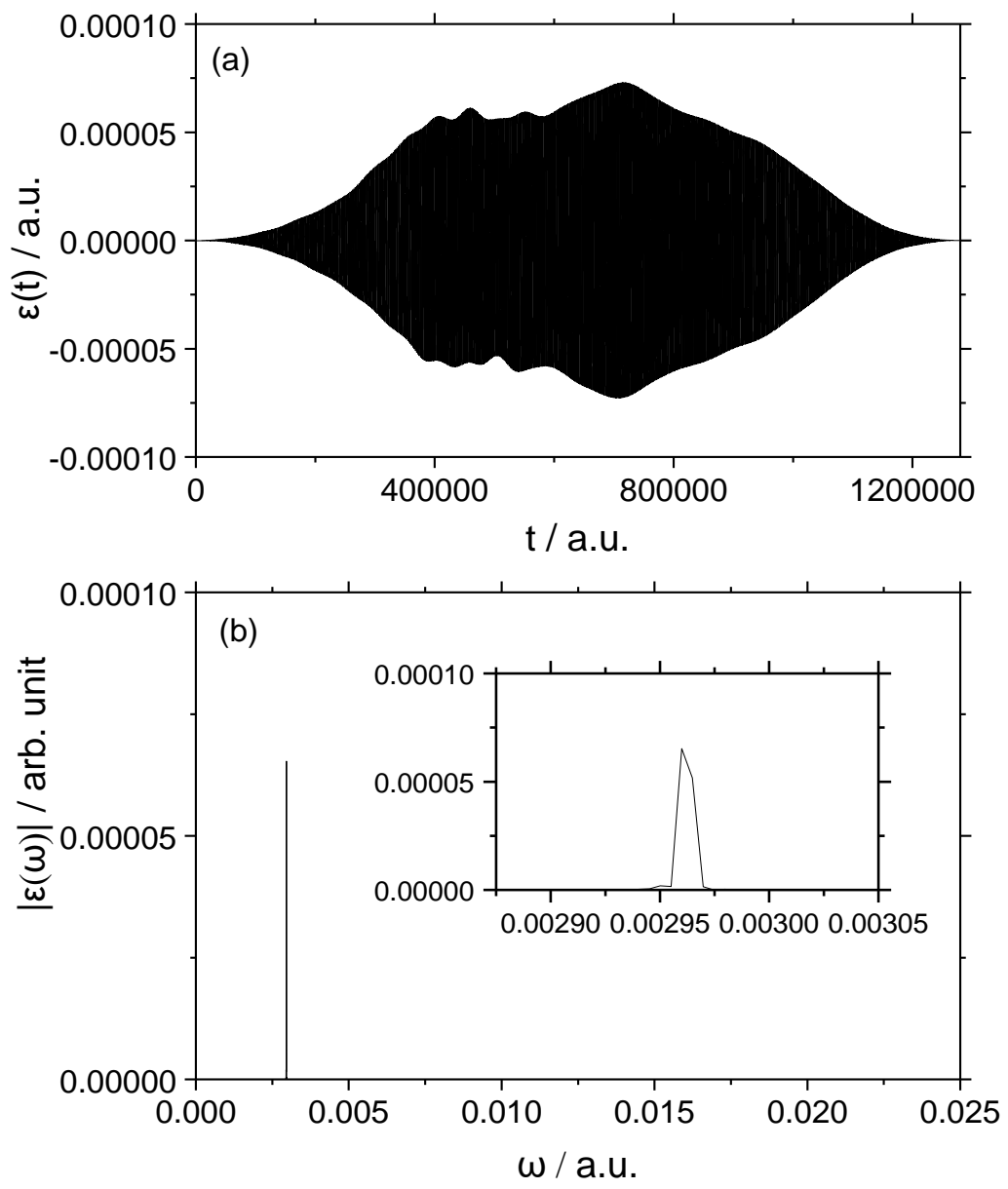

Fig. 11 

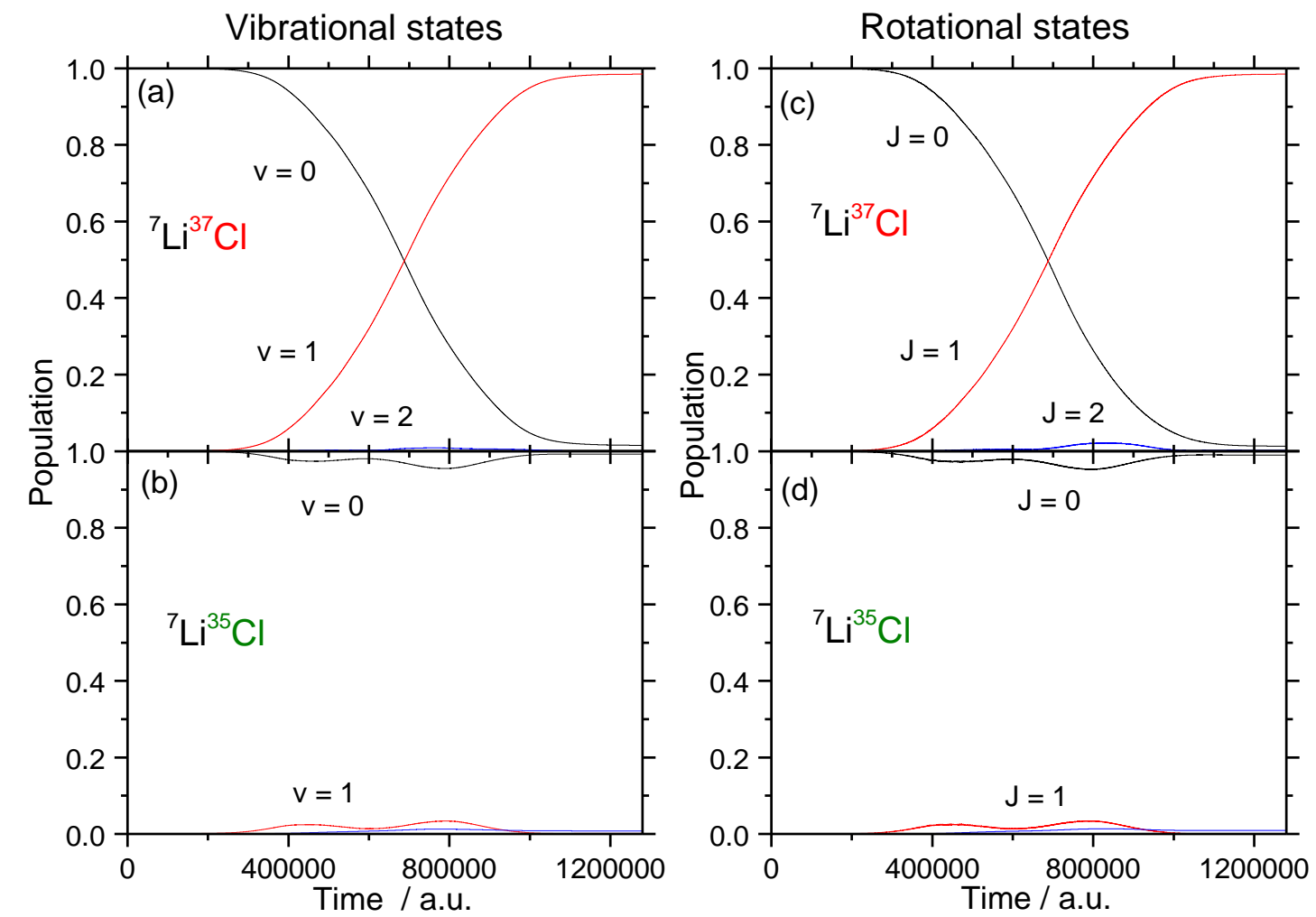

Fig. 12 

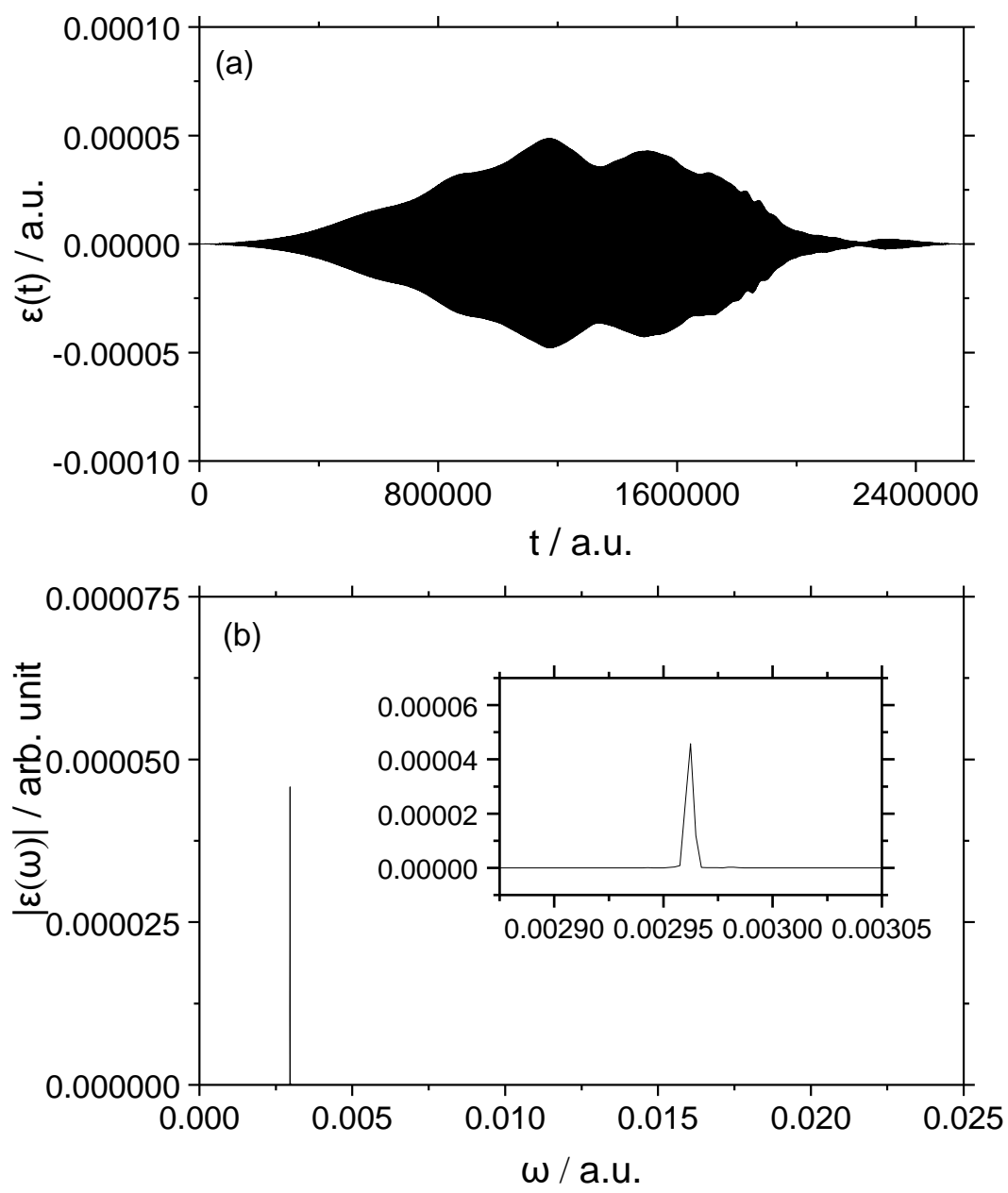

Fig. 13 
Vibrational states

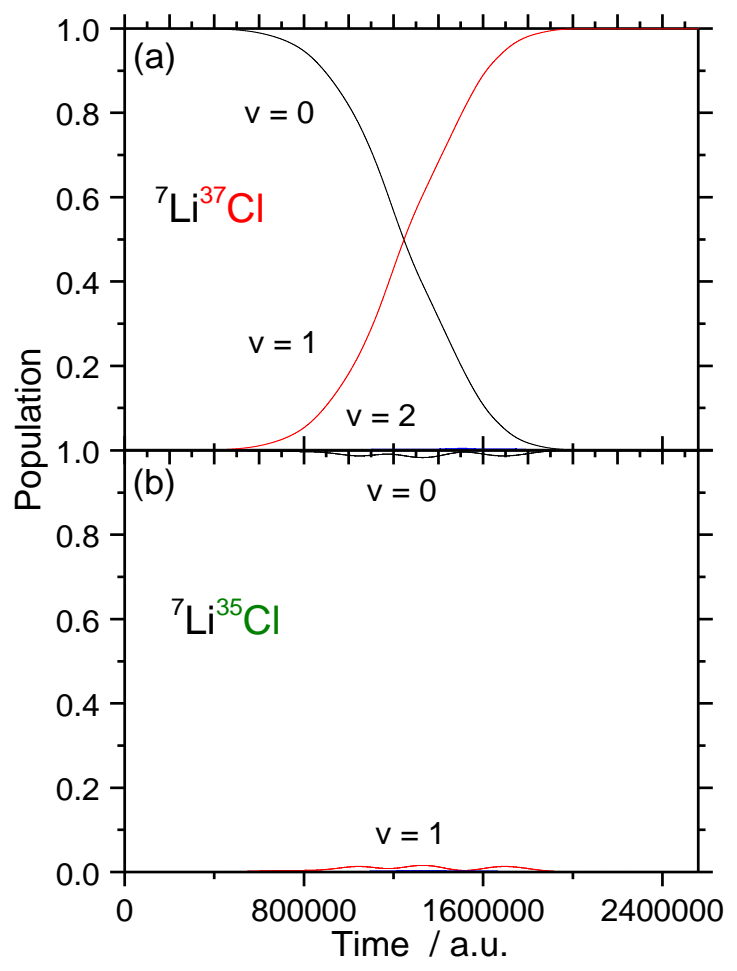

Rotational states

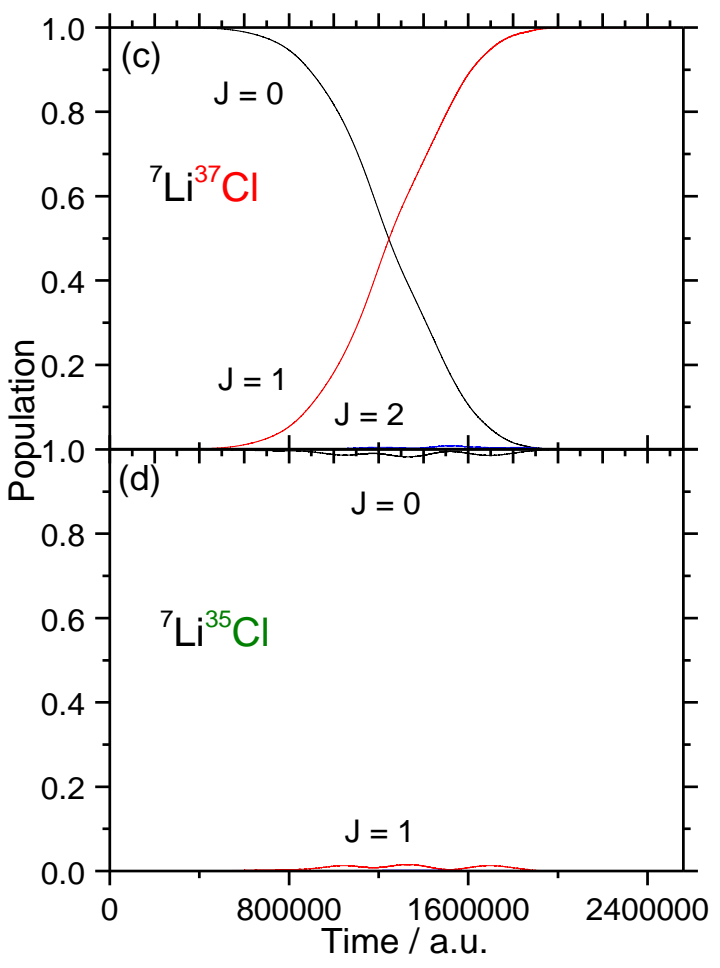

Fig. 14 